\title{
PROBING HIGH-REDSHIFT GALAXY FORMATION AT THE HIGHEST LUMINOSITIES: NEW INSIGHTS FROM DEIMOS SPECTROSCOPY
}

\author{
Kyoung-Soo Lee ${ }^{1}$, Arjun Dey ${ }^{2}$, Michael C. Cooper ${ }^{3}$, Naveen Reddy ${ }^{4}$, Buell T. Jannuzi ${ }^{5}$
}

\begin{abstract}
We present Keck DEIMOS spectroscopic observations of the most UV-luminous star-forming galaxies at redshifts $3.2<z<4.6$. Our sample, selected in the Boötes field of the NOAO Deep Wide-Field Survey, contains galaxies with luminosities of $L^{*} \lesssim L_{\mathrm{UV}} \lesssim 7 L^{*}$ and is one of the largest samples to date of the most UV-luminous galaxies at these redshifts. Our spectroscopic data confirm 41 candidates as star-forming galaxies at $3.2<z<4.6$ and validate the relatively clean selection of the photometric candidates with a contamination rate of $11 \%-28 \%$. We find that the fraction of Ly $\alpha$ emitting galaxies increases with decreasing UV luminosity. None of the 12 galaxies with $M_{\mathrm{UV}}<-22$ (i.e., $L_{\mathrm{UV}}>3 L^{*}$ ) exhibit strong Ly $\alpha$ emission. We find strong evidence of large-scale outflows, transporting the neutral/ionized gas in the interstellar medium away from the galaxy. Galaxies exhibiting both interstellar absorption and Ly $\alpha$ emission lines show a significant offset between the two features, with the relative velocity of $200-1150 \mathrm{~km} \mathrm{~s}^{-1}$. We find tentative evidence that this measure of the outflow velocity increases with UV luminosity and/or stellar mass. The luminosity- and mass-dependent outflow strengths suggest that the efficiency of feedback and enrichment of the surrounding medium depend on these galaxy parameters. We also stack the individual spectra to construct composite spectra of the absorption-line-only and Ly $\alpha$-emitting subsets of the UV luminous galaxies at $z \simeq 3.7$. The composite spectra are very similar to those of lower-redshift and lower-luminosity LLyman break galaxy (LBG) samples, but with some subtle differences. Analyses of the composite spectra suggest that the UV luminous LBGs at $z \simeq 3.7$ may have a higher covering fraction of absorbing gas, and may be older (or have had more prolonged star formation histories) than their lower-redshift and lower-luminosity counterparts. In addition, we have discovered that five galaxies in the sample belong to a massive overdensity at $z=3.78$. Finally, two galaxies each show two distinct sets of interstellar absorption features. The latter may be a sign of a final stage of major merger, or clumpy disk formation. These systems are not expected in our sample: their presence implies that frequency of such sources among our luminous $z \simeq 3.7$ LBGs may be an order of magnitude higher than in lower redshift and lower luminosity samples.
\end{abstract}

Subject headings: cosmology:observations - galaxies:distances and redshifts - galaxies:evolution galaxies:formation

\section{INTRODUCTION}

Deep multi-wavelength space- and ground-based imaging surveys have enabled the identification and study of large numbers of high-redshift galaxies (e.g., Steidel et al. 2003, 2004; Giavalisco et al. 2004b). These studies have made significant strides in characterizing various global statistics of the high-redshift galaxy population, including their UV luminosity function, stellar mass function, and clustering properties (e.g., Steidel et al. 1999; Giavalisco et al. 2004a; Bouwens et al.|2007; Reddv \& Steidel|2009; Ouchi et al. 2004a,b; Lee et al. 2006, 2009, 2012b; González et al. 2011). In addition, the overall distribution of dust content, stellar population ages, and sizes have been determined at different cosmic epochs (Ferguson et al. 2004; Bouwens et al. 2004, 2009, 2012; Stark et al. 2009; Reddy et al. 2006, 2012a; Lee et al. 2012a; Finkelstein et al. 2012).

\footnotetext{
${ }^{1}$ Department of Physics, Purdue University, 525 Northwestern Avenue, West Lafayette, IN 47907

${ }^{2}$ National Optical Astronomy Observatory, Tucson, AZ 85726

${ }^{3}$ Department of Physics and Astronomy, University of California, Irvine, CA 92697

${ }^{4}$ Department of Physics and Astronomy, University of California, Riverside, 900 University Avenue, Riverside, CA 92521

${ }^{5}$ Steward Observatory, University of Arizona, Tucson, AZ 85721
}

Nevertheless, several questions remain about the origin and evolution of these galaxies. For example, what physical processes govern the star-formation histories of these galaxies? Do the star-formation, assembly and chemical evolutionary histories vary as function of galaxy luminosity, halo and stellar mass, and environment? If they do, what drives these differences?

While useful constraints have been placed on some of these questions based on statistical studies (e.g., Steidel et al. 2005; Lee et al. 2011, 2012b; Papovich et al. 2011; Reddy et al. 2012b), the deep spectroscopy necessary to measure the age and metallicity of the stellar populations, interstellar abundances, and large-scale outflows, has been lacking (but see Giavalisco 2002; Shapley 2011, for review). The main challenge has been the faintness of most high-redshift galaxies, which precludes high signal-to-noise ratio $(\mathrm{S} / \mathrm{N})$ spectroscopic studies. The usefulness of deep spectroscopy in shedding light on the chemical, kinematic, and geometrical details within the galaxy has been amply demonstrated in a few studies of very rare, bright galaxies (either intrinsically luminous or gravitationally amplified; e.g., MS1512cB58: Pettini et al. 2000, 2002; Steidel et al. 2010), and also by studies of high $\mathrm{S} / \mathrm{N}$ composite spectra created by averaging many low-S/N spectra (e.g., Shapley et al. 2003; Steidel et al. 2001, 2010; Jones et al. 2012). These 
pioneering studies have shown that galactic outflows are powerful enough to drive out as much gas as that consumed by the star-formation at velocities close to the escape velocity, thereby effectively enriching the circumgalactic medium. Shapley et al. (2003) showed that the interstellar medium (ISM, hereafter) in these galaxies must be clumpy based on the observed anti-correlation between the $\operatorname{Ly} \alpha$ and interstellar line equivalent widths. When a galaxy is observed through optically thinner regions, more Ly $\alpha$ photons escape while less interstellar absorption will occur.

Most of these results pertain to galaxies in a relatively narrow range of UV luminosity $\left(\approx L^{*}\right)$ and redshift (typically $z \simeq 2-3$ ). This luminosity and redshift ranges represent a "sweet spot" for efficient studies of relatively large numbers of UV-luminous galaxies, since the space density of high-redshift galaxies falls precipitously at $L \gtrsim L^{*}$ while the success rate of spectroscopic identification declines to lower luminosities and higher redshifts. However, several studies have begun to suggest that galaxies of different luminosities may exhibit different characteristics (e.g., the fraction of Ly $\alpha$ emitting galaxies or the stellar-mass-to-UV-light ratio: see Stark et al. 2010; Lee et al. 2012b). It is therefore important to investigate the full luminosity range of high-redshift galaxies and determine how representative the current physical picture, offered by spectroscopic efforts of $L \gtrsim L^{*}$ galaxies, is to more- or less-luminous galaxies and/or at higher redshift. Widening the dynamic range will also improve our understanding of the physical processes of the feedback and mass assembly histories within different dark matter halo environments (e.g., Ouchi et al. 2004b; Lee et al. 2006; Hildebrandt et al. 2007).

In this paper, we present a pilot study of spectroscopic observations of higher-redshift $(3.2<z<4.6)$, higherluminosity $\left(L^{*} \lesssim L \lesssim 7 L^{*}\right)$ galaxies with the primary aim of critically assessing our current understanding of galaxy formation at high redshift.

We use the WMAPr cosmology $\left(\Omega, \Omega_{\Lambda}, \sigma_{8}, h_{100}\right)=$ $(0.27,0.73,0.8,0.7)$ Komatsu et al. 2011). Adopting the WMAP9 or Planck cosmology (Hinshaw et al. 2012; Planck Collaboration et al. 2013, respectively) would change angular sizes or luminosities by less than $1 \%$. Magnitudes are given in the AB system (Oke \& Gunn 1983) unless noted otherwise.

\section{DATA AND PHOTOMETRIC SELECTION OF GALAXIES} $\mathrm{AT} Z \simeq 3.7$

Our photometric candidates are selected from the Boötes field of the NOAO Deep Wide-Field Survey (NDWFS; Jannuzi \& Dey 1999). Briefly, the NDWFS $B_{W} R I$ band data were obtained using the Mosaic camera on the Mayall $4 \mathrm{~m}$ Telescope, and consist of 27 separate pointings covering a contiguous $9.3 \mathrm{deg}^{2}$ area.

For the spectroscopic target selection, we focused on three pointings obtained under the best observing conditions. We chose pointings where: (1) all three photometric bands have seeing better than $1^{\prime \prime}$ and within $0.1^{\prime \prime}$ of one another; and (2) the $50 \%$ completeness limit in the three bands are deeper than $B_{W}>26.8, R>25.8$,

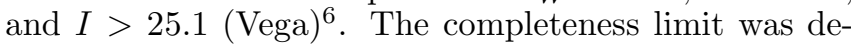
termined by adding artificial stellar objects (convolved

6 The NDWFS images are calibrated in Vega magnitudes. The correction to be applied to convert to the AB system is 0.019,

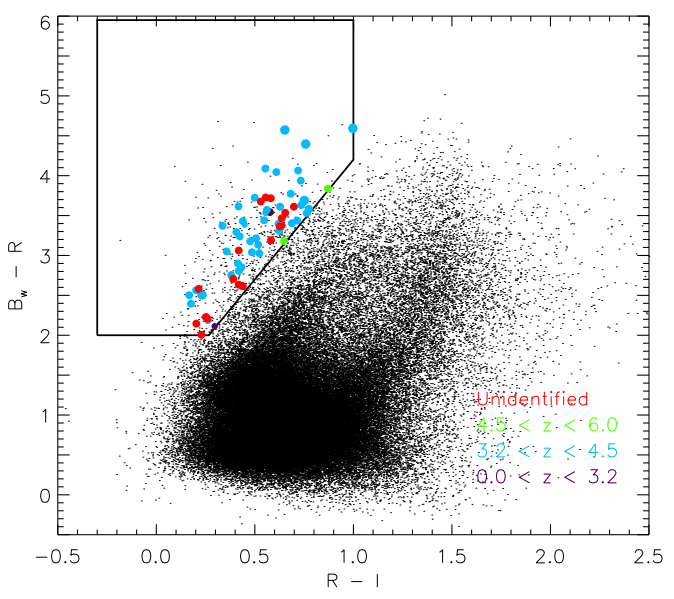

FIG. 1.- Locations of the NDWFS sources on the $B_{W}-R-R-I$ plane are shown together with the Lyman-break color selection criteria outlined by the black solid line. The colors are based on Vega magnitudes. The spectroscopic targets are overlaid in color showing the interlopers ( $z<3.2$; dark purple), LBGs at $3.2<z<$ 4.2 (blue), LBGs at $z>4.2$ (green), and the sources that could not be identified (red).

with a Moffat profile matched to the measured seeing) to the images and recovering them with SExtractor using the identical parameters as the real data (tabulated on the survey web page). ${ }^{7}$ These requirements allow robust photometric color selection without degrading the images by convolving them to a common seeing, and a maximum surface density within the multi-object masks down to $I \approx 25.0$.

Our photometric sample is selected by applying a Lyman-break color-selection technique to the $B_{W} R I$ data (e.g., Steidel et al. 1999; Giavalisco et al. 2004a; Bouwens et al. 2007). The Lyman-break technique is designed to identify a UV bright star-forming galaxy (or Lyman break galaxy, LBG, hereafter) with a strong spectral break (at a rest-frame wavelength $\lambda \leq 1216 \AA$ ) resulting from absorption by the intervening $\operatorname{Ly} \alpha$ forest. At $3.3<z<4.3$, this break lies between the $B_{W}$ and $R$ bands. The color criteria (in Vega magnitudes) used to select the candidates are identical to that used for a larger sample of photometric candidates described in Lee et al. (2011).

$$
\begin{array}{ccc}
\left(B_{W}-R\right) & \geq 1.2+3.0 \times(R-I) & \left(B_{W}-R\right) \geq 2.0 \\
(R-I) & \geq-0.3 \quad \mathrm{~S} / \mathrm{N}(\mathrm{R}) \geq 3 & \mathrm{~S} / \mathrm{N}(\mathrm{I}) \geq 7
\end{array}
$$

The total number of sources satisfying the color selection criteria are 738. In Figure 1, we show the color locations of all sources together with the selection window. The spectroscopic targets are shown as colored dots; these are discussed further in 3 .

\section{KECK DEIMOS OBSERVATIONS}

Spectroscopic observations of our LBG candidates were carried out on the night of May 102010 using the DEep Imaging Multi-Object Spectrograph (DEIMOS) on the 10-m Keck II telescope (Faber et al. 2003). We used 6000 line $\mathrm{mm}^{-1}$ grating blazed at $7500 \AA$, yielding a

0.215 , and $0.459 \mathrm{mag}$ for the $B_{W}, R$ and $I$-band, respectively; e.g., $I_{\mathrm{AB}}=I_{\text {Vega }}+0.459$.

7 http://www.noao.edu/noao/noaodeep/DR3/dr3-descr.html 

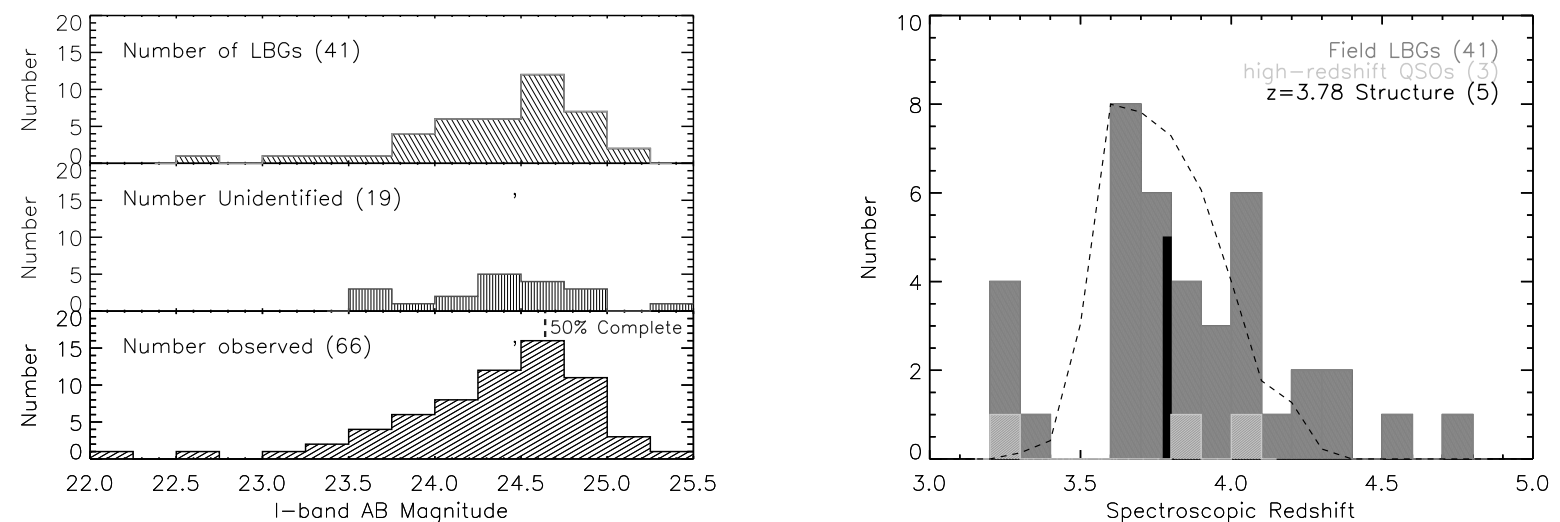

FIG. 2.- Left: the magnitude distribution of our targets (66), confirmed LBGs (41) at $z=3.2-4.7$, and unidentified sources (20) are shown as a function of $I$-band magnitude. The magnitude at which the source detection is $50 \%$ complete is indicated as dashed line on top. Right: the redshift distribution of the spectroscopically confirmed LBGs and QSOs in the NDWFS Boötes field. Also shown are the redshift distribution of three QSOs and five galaxies in the overdensity at $z=3.78$. The expected redshift distribution from our photometric simulations is shown in dotted line.

spectral coverage of $4300-9600 \AA$ at the dispersion of $0.65 \AA \mathrm{pixel}^{-1}$.

We designed three masks (BDm_01, BDm_02, and BDm_03) covering a total of 66 candidates. The mask locations were selected with the aim of maximizing the number of LBG candidates within each DEIMOS field of view. The photometric candidates were put in two categories as "primary" (S/N(I) $\geq 10)$ and "secondary" $(7 \leq \mathrm{S} / \mathrm{N}(\mathrm{I})<10)$ targets, with the primary targets driving the DEIMOS field placement. Table 1 summarizes the basic properties of each mask. The slit width was $1^{\prime \prime} .2$ in all three masks. The effective spectral resolution estimated from the sky lines is $3.2 \AA$. Each mask was observed with $2.0-2.5 \mathrm{hr}$ in good conditions, with seeing between $0^{\prime \prime} .6$ and $0^{\prime \prime} .8$ throughout the night.

The data were reduced using the spec2d IDL pipeline developed for the DEIMOS instrument (Newman et al. 2012; Cooper et al. 2012). The wavelength calibration was carried out using observations of $\mathrm{Ne}+\mathrm{Ar}+\mathrm{Kr}+\mathrm{Xe}$ arc lamps. The two-dimensional sky-subtracted spectra were inspected visually to validate the automatic extraction of the one-dimensional spectra by the DEIMOS pipeline. There are a few cases where another galaxy fell into the slit and affected the sky subtraction, or the galaxy was located too close to the edge of the slit. In these cases, re-extraction using the IRAF routine noao.twodspec.apall significantly improved the quality of the one-dimensional spectra.

Redshifts were determined by visual examination from both one- and two-dimensional spectra. We used the SpecPro software (Masters \& Capak 2011) to check the spectra simultaneously with the available imaging data. Redshift identification is made by looking for the presence of Ly $\alpha$ emission, interstellar absorption lines, and the continuum break due to the Lyman alpha forest. Of the $66 B_{W}$-band dropout candidates observed during the run, we have determined redshifts for 46 sources. In Table 2, we list the basic properties of the 46 galaxies/QSOs including their coordinates, redshifts, and magnitudes.

Of the 46 spectroscopic identifications, 41 sources are galaxies at the expected redshift range, 2 are low-redshift interlopers at $z=0.373$ and 0.631 (identified via [O II] 3727 and $\mathrm{H} \alpha$ emission lines), and 3 are QSOs at $z=$ $3.289,3.835,4.041$. Of the remaining 20 sources for which we were unable to determine redshifts, 2 show feature- less red continua, indicating that they are likely dusty galaxies at $z<2$. An additional 5 galaxies show a clear continuum break consistent with being an LBG, but the $\mathrm{S} / \mathrm{N}$ is too poor to identify emission or absorption features to confirm (or measure precise) redshifts. Finally, no information could be obtained on 2 sources because the slitlet was contaminated and therefore masked out during the reduction. Hence, the estimated contamination rate from the spectroscopic identifications is $11 \%$ (5/46), where we have included the $3<z<4$ QSOs as contaminants. The lower limit on the contamination rate for the full sample is also $11 \%(=(5+2) /(66-2))$ where the total of 64 galaxies excludes the two sources which were masked out. In the most pessimistic case where all of the unidentified sources are not LBGs, the contamination rate is $28 \% ; 1-(41+5) /(66-2)$. The magnitude distributions of our targets, confirmed LBGs, and unidentified sources are presented in Figure 2 (left).

For the majority of confirmed LBGs (36/41; i.e., 88\%), a clear UV continuum and at least two interstellar lines were detected with sufficient $\mathrm{S} / \mathrm{N}$ to determine interstellar redshifts (Table 21). The remaining 5 LBGs are identified only by their Ly $\alpha$ emission. Of those 5 , we detect a UV continuum in three galaxies but at a low $\mathrm{S} / \mathrm{N}$ that we could not measure interstellar redshifts. Therefore, the confirmed LBGs at $z \leq 4.2$ represent approximately a (continuum-)flux-limited sample minimally affected by the spectroscopic bias which preferentially identifies line emitters.

Figure 2 (right) shows the redshift distribution of all spectroscopically identified sources at $z>3$, including 41 LBGs and 3 high-redshift QSOs. The observed highredshift tail (at $z>4.2$ ) can be explained by the spectroscopic selection effect that favors redshift identification for Ly $\alpha$ emitting sources. Of the seven sources at $z>4.2$, four are secondary targets, detected at a low $\mathrm{S} / \mathrm{N}$ in the $I$-band, and five are observed with a high Ly $\alpha$ rest-frame EW $\left(W_{0} \geq 18 \AA\right)$. The average EW for those five sources is $W_{0}=24.4 \AA$, much higher than that measured for the full sample $\left(W_{0}=-1.2 \AA\right)$. Among the measured redshifts are five galaxies lying within a small redshift range $(z=3.782-3.787)$ and within $1.5^{\prime}$ from each other (all in the mask BDm_02). The close physical proximity of these five galaxies strongly suggests that they belong to 
TABLE 1

Summary of DEIMOS Multi-OBJect Slit Masks

\begin{tabular}{ccccccc}
\hline \hline Mask & Field $^{\mathrm{a}}$ & R.A. $^{\mathrm{b}^{3}}$ & Decl. $^{\mathrm{b}}$ & $N_{\text {primary }}$ & $N_{\text {secondary }}$ & $t_{\text {exp }}(\mathrm{hr})$ \\
\hline BDm_01 & NDWFSJ1426p3236 & $14: 27: 10.30$ & $32: 34: 11.0$ & 20 & 9 & 2.50 \\
BDm_02 & NDWFSJ1431p3236 & $14: 31: 26.41$ & $32: 25: 40.8$ & 14 & 0 & 2.25 \\
BDm_03 & NDWFSJ1437p3347 & $14: 37: 34.37$ & $33: 36: 36.1$ & 16 & 7 & 1.99 \\
All & - & - & - & 50 & 16 & \\
\hline
\end{tabular}

a The name of the fields in the NDWFS survey Web site

b The J2000 coordinates of the center of each pointing

TABLE 2

The Summary of the Spectroscopic Sample

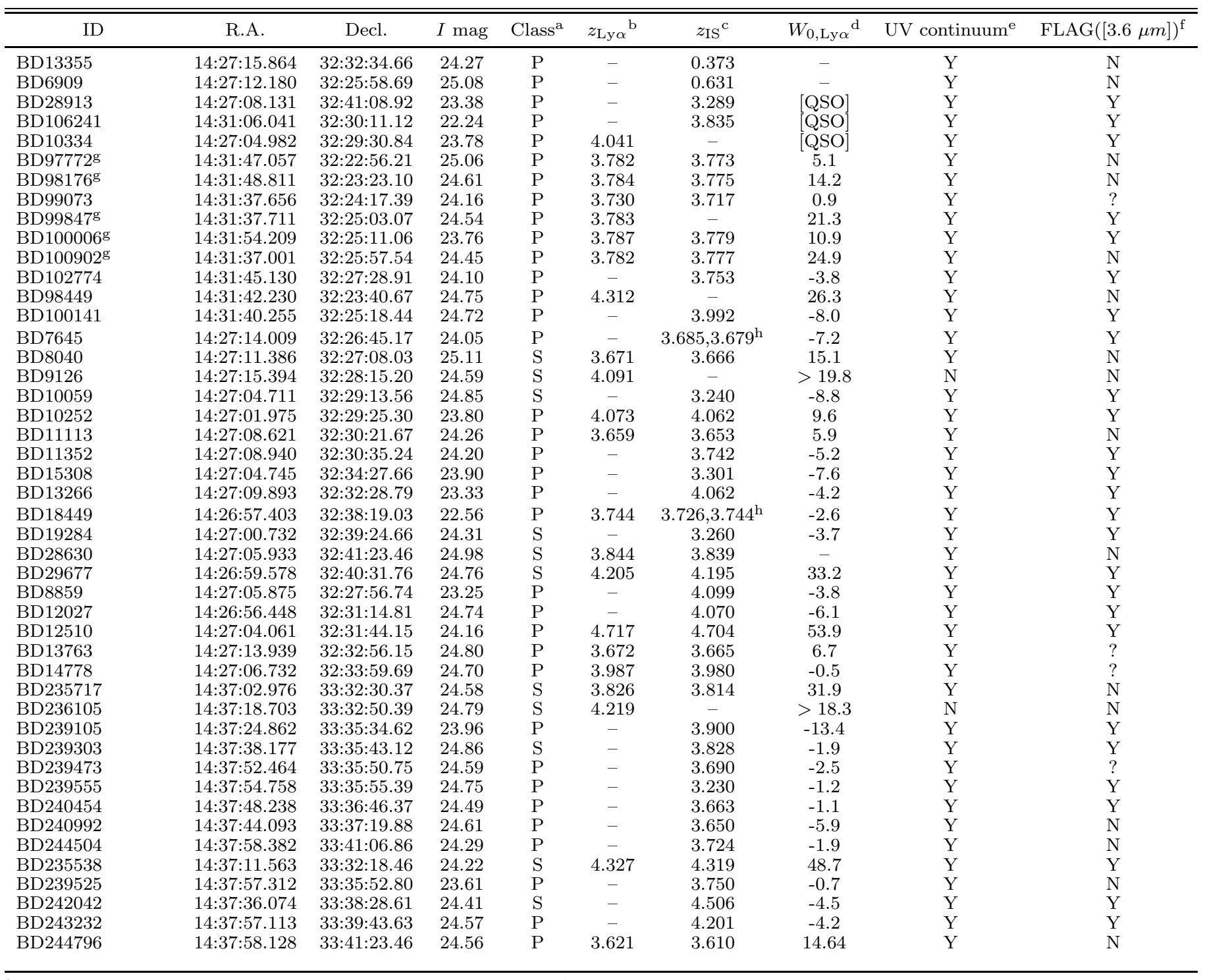

a "P" is for primary and " $\mathrm{S}$ " is for secondary targets, based on the $\mathrm{S} / \mathrm{N}$ in the detection band

b The Ly $\alpha$ redshift is determined from the peak of the Ly $\alpha$ emission

c The interstellar redshift is determined from the peak of the interstellar absorption line

$\mathrm{d}$ The rest-frame equivalent width of $\operatorname{Ly} \alpha$, only measured for LBGs

e The UV continuum detected in the spectrum?

f Spitzer IRAC [3.6 $\mu \mathrm{m}$ ] band detection: "Y" for detected, "N" for undetected, and "?" for uncertain

$\mathrm{g}$ The galaxies in the $z=3.78$ structure (see 5.1

$\mathrm{h}$ The galaxies with multiple interstellar redshifts (see 5.2 ) 


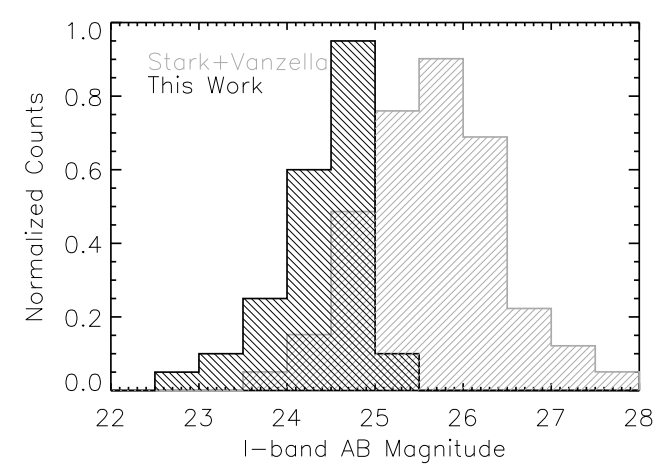

FIG. 3.- The normalized I-band magnitude distribution of our sample is compared to that of the Vanzella+Stark sample (Vanzella et al. 2009; Stark et al. 2010). The galaxies in our sample are roughly 2.1 times more UV-luminous than those in the Vanzella+Stark sample.

a massive structure (see \$5.1). In consideration of these factors, the observed $N(z)$ is in a reasonable agreement with the redshift distribution expected from photometric simulations as shown in Figure 2 (dotted line in the right panel; see Lee et al. 2011 for the details of the simulations), i.e., that our selection criteria yield a galaxy population at $z \simeq 3.7 \pm 0.4$.

\section{THE PHYSICAL PROPERTIES OF MOST UV-LUMINOUS LBGS}

The spectra provide rich details about the physical properties of the most UV-luminous galaxies and show striking differences in the galaxy properties at different UV luminosities (i.e., star-formation rates). Before we proceed, it is useful to compare our sample to other large spectroscopic samples (generally of less UVluminous galaxies) at the same redshift. Vanzella et al. (2006, 2009) observed $\approx 50$ galaxies at $z \simeq 3.7$ with the FORS2 spectrograph on the Very Large Telescope. More recently, Stark et al. (2010) obtained a large sample $(\approx 120)$ of galaxies at $z=3.0-4.5$ with the DEIMOS on the Keck II Telescope. Both samples were drawn from the same photometric candidates selected from the Great Observatories Origins Deep Survey (Giavalisco et al. 2004b), using the Lyman break technique described in $\S 2$. In Figure 3, we compare the magnitude distribution of our sample to those of the combined Vanzella+Stark samples. The difference in the median luminosities between the two samples is 0.8 mag. As the redshift selection functions for these samples are nearly identical, the median UV luminosity of our sample is roughly 2.1 times higher than that of the Vanzella+Stark samples.

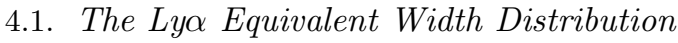

We measure the Ly $\alpha$ EW for each galaxy directly from the one-dimensional spectra. The average continuum level $c_{\text {red }}$ is determined by taking a weighted average of the pixels in the region corresponding to the rest-frame $1225-1255 \AA$ (and excluding all pixels affected by $\mathrm{OH}$ sky lines). The line flux $F_{\mathrm{Ly} \alpha}$ is computed by summing the flux in excess of the average continuum level $c_{\text {red }}$ at $\lambda_{\text {rest }}=1213-1221 \AA$, and the rest-frame EW $W_{0}$ is then estimated as $W_{0} \equiv F_{\text {Ly } \alpha} /\left[c_{\text {red }}(1+z)\right] \Delta \lambda$. Positive $W_{0}$ represents emission. Random errors in the EWs are estimated from the uncertainties in the line flux and continuum level.

In Figure 4, we show the distribution of the rest-frame Ly $\alpha$ EWs for all LBGs in our sample. Even though a half of our sample exhibit some level of Ly $\alpha$ in emission, the median EW is negative. For the 36 "field" galaxies, the median $W_{0}$ is $-1.2 \AA$. On the other hand, the 5 "protocluster" galaxies appear to have a much higher median EW of $14.2 \AA$. There are only $7 / 41(17 \%)$ galaxies that $W_{0} \geq 20 \AA$ in our sample. Of those 7 galaxies, 4 are at $z>4.2$ and additional 2 belong to the $z=3.78$ structure (right panel of Figure 4). If we accept the possibility that the majority of $z>4.2$ galaxies are selected because of their strong Ly $\alpha$ emission, and that the galaxies in the $z=3.78$ structure are not representative of the remaining $(z<4.2)$ field sample, then only $1 / 32$ "field galaxies" $(3 \%)$ have $W_{0} \geq 20 \AA$ (BD235717, $W_{0}=32 \AA$ ). These estimates are consistent with the measurements made by Stark et al. (2010, see their Figure 13) and extend these to higher UV luminosities.

To investigate the frequency of high-EW sources with UV luminosity, we compute the luminosity of the sources at the rest-frame $1700 \AA$ (Figure 4, right). First, we adopted the Madau (1995) prescription and calculated the effective flux attenuation by the intergalactic medium (IGM) in the $R$-band as a function of redshift, which changes from $\Delta m=0$ at $z<3.6$, to $\Delta m=0.17$ at $z=4$, to $\Delta m=0.39$ at $z=4.3$. Second, from the IGMcorrected $(R-I)$ color, we estimate the UV spectral slope $\beta$ assuming a power-law spectral energy distribution (i.e., $\left.f_{\lambda} \propto \lambda^{\beta}\right)$. The absolute magnitude at $\lambda=1700 \AA, M_{1700}$, is computed by interpolating from the $I$-band total magnitude. For comparison, we also show the locations of the $B$ dropouts in the GOODS South (Vanzella et al. 2009) as open triangles in the same figure. We computed the UV magnitudes at $1700 \AA$, by interpolating from the $(i-z)$ color, both of which are free of intergalactic absorption at this redshift range. We note that Vanzella et al. (2009) did not measure the EWs for the galaxies with no Ly $\alpha$ emission, and therefore the triangle points at zero EW should be considered as an upper limit.

To understand the spectroscopic bias, which preferentially selects strong Ly $\alpha$ line emitters, we computed the rest-frame EWs corresponding to the $10 \sigma$ limiting line flux $\left(\sim 1.8 \times 10^{-17} \mathrm{erg} \mathrm{cm}^{-2} \mathrm{~s}^{-1}\right)$ at different continuum luminosities at rest-frame $1700 \AA$ (dashed line). We assumed the spectral slope $\beta=-1.6$, consistent with the average value measured at this luminosity range (Lee et al. 2011). Also shown in Figure 4 (right panel) as vertical dashed line is the $50 \%$ completeness limit in $I$-band source detection based on the image simulations we carried out on these fields. These considerations suggest that our data allow to identify line emission at the $10 \sigma$ level for galaxies with $\mathrm{EW} \gtrsim 10 \AA$ at the faint end of our sample.

Due to the small sample size, it is difficult to draw a conclusion based on our sample alone. However, the picture becomes clearer when we consider the Vanzella sample and ours jointly. The observations of the former should be at least as sensitive as our observations, and therefore their $10 \sigma$ limit should be comparable to ours (dashed line in Figure 4 right). From the combined sample, the increasing frequency of higher-EW sources 

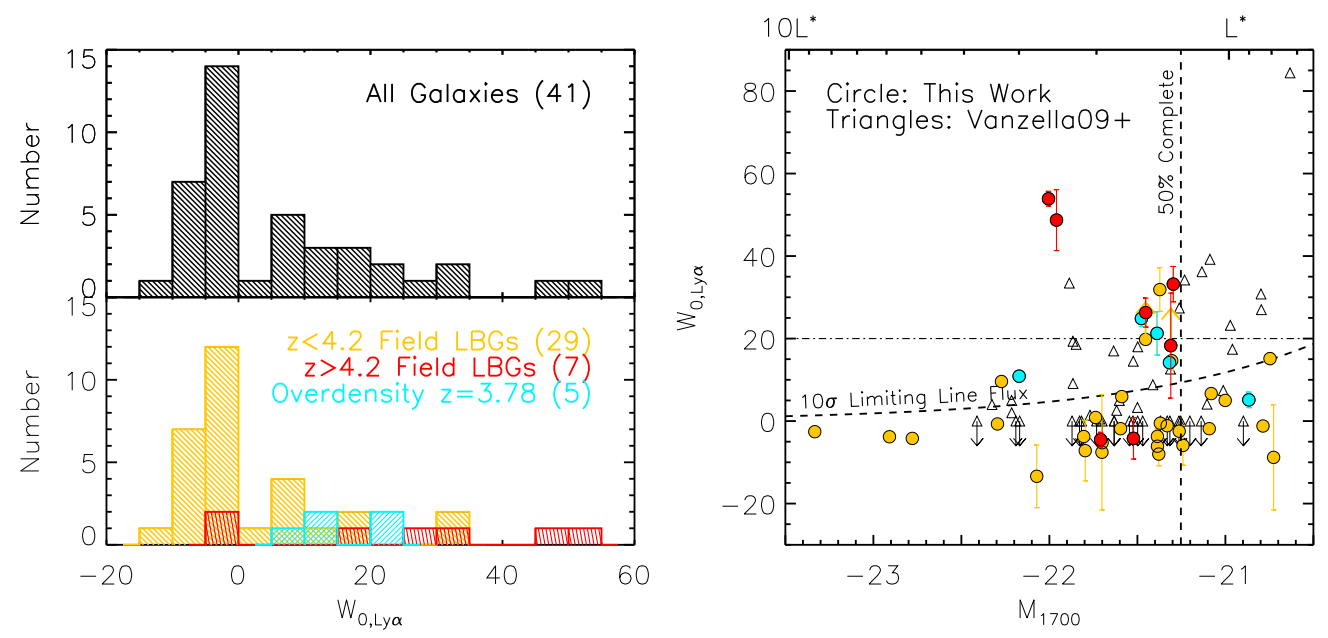

FIG. 4.- Left: The distribution of the rest-frame Ly $\alpha$ EWs for the entire spectroscopic sample (top panel) and separated into the 5 galaxies in the $z=3.78$ structure and the 36 "field" galaxies (bottom panel). The difference in the median values $\left(W_{0}=14.2 \AA\right.$ and $-1.2 \AA$, respectively) between the two samples is significant. Right: The distribution of the Ly $\alpha$ EWs as a function of UV luminosity suggests a lack of strong Ly $\alpha$ emitters at the very bright end. The majority of the high-EW sources are at the high-redshift tail of our selection function, i.e., at $z>4.2$, which is likely a result of the spectroscopic selection bias. On the other hand, the relatively strong Ly $\alpha$ emission from the $z=3.78$ structure appears to be real, with $4 / 5$ sources observed with $W_{0}>10 \AA$. When $z>4.2$ galaxies and those at $z=3.78$ are discarded, only $1 / 29$ (i.e., $3 \%$ ) galaxies have $W_{0} \geq 20 \AA$.

(i.e., those with stronger Ly $\alpha$ emission) toward faint luminosities is evident. Interestingly, we find that there are no strong Ly $\alpha$ emitters (i.e., with $W_{0}>10 \AA$ ) at $M_{\mathrm{UV}}<-22.2\left(L_{\mathrm{UV}} \geq 3 L_{z \simeq 3.7}^{*}\right)$ in the combined sample even though there is no selection bias against identifying such sources. The lack of very bright, high-EW sources at $z \sim 4$ appears to be in conflict with that found at $z \sim 6.0-6.5$ (Curtis-Lake et al. 2012). While reversal of such a trend could be potentially interesting, better statistics are needed to place stronger constraints on the Ly $\alpha$-emitting fraction at the brightest end.

\subsection{Large-scale Outflows}

Interstellar absorption lines that are blue-shifted with respect to the galaxy's systemic redshift are frequently observed in local and high-redshift starforming galaxies over a wide range of luminosities and masses. These observations provide strong evidence of galactic-scale outflows that remove a considerable amount of neutral and ionized gas from the galaxy (Heckman et al. 2000; Martin 2005, 2006; Schwartz \& Martin 2004; Shaplev et al. 2003; Rupke et al.|2005; Adelberger et al.|2005; Vanzella et al. 2009; Weiner et al. 2009; Steidel et al. 2010). The quantity of material ejected from a galaxy into the IGM and the physical process(es) responsible are critical to our understanding of "feedback", which is thought to shape a galaxy's star-formation, chemical and evolutionary history, as well as the chemical history of the IGM. In particular, the relative efficiency of the feedback in galaxies of different luminosities/masses can shed light on their respective assembly histories. Martin (2005) and Weiner et al. (2009) observed that the outflow velocity increases with the SFRs of the galaxy (local ultraluminous IR galaxies for the former, and $z \simeq 1.4$ starforming galaxies for the latter; also see Rupke et al. 2005). On the other hand, several more recent studies of local and intermediate-redshift galaxies do not find such a strong trend (Chen et al. 2010; Kornei et al. 2012), and the question regarding the luminosity dependence of feedback remains debated.

At high redshift, measurements of outflows based on the largest sample of star-forming galaxies at $z \simeq 2-3$ do not show any evidence of a luminosity dependence (Steidel et al. 2010) and instead find marginal evidence for the opposite trend, i.e., that more massive galaxies have a lower outflow velocities as traced by the centroids of interstellar absorption lines (see Figure 3 of Steidel et al. 2010). The discrepancy between studies at different redshifts is hard to understand, but may be in part explained by large scatter inherent to the measured outflow velocities, which sensitively depends on the viewing angle of the observer as well as the opening angle of the winds with respect to the galactic axis. Furthermore, different line diagnostics studied at different redshifts may trace different kinematic components in the ISM, making direct comparison challenging. For example, the $\mathrm{Na} \mathrm{D}$ doublet (arising in neutral gas) is used to trace the outflowing gas in local galaxies, whereas intermediate redshift studies use Fe II, Mg II, Mg I, which generally have higher ionization potentials. Furthermore, galaxies at lower redshift can be (and typically are) observed at a higher spectral resolution, where the systemic and outflowing components can be more easily separated (e.g., Chen et al. 2010); this approach is impractical for faint, high-redshift galaxies. Finally, the limited dynamic range in galaxy properties in most highredshift samples studied to date may also play a role in erasing any trend of outflow velocity with other galaxy properties, especially given the inherently large scatter in the measurements or in the intrinsic distributions. Indeed, the local correlation is largely driven by the measurements of dwarf starbursts and (Ultra-)luminous IR galaxies (Schwartz \& Martin 2004; Martin 2005). In this section, we attempt to improve on previous studies of outflows by extending the investigation to the more luminous high-redshift sample of galaxies uncovered by our survey. 

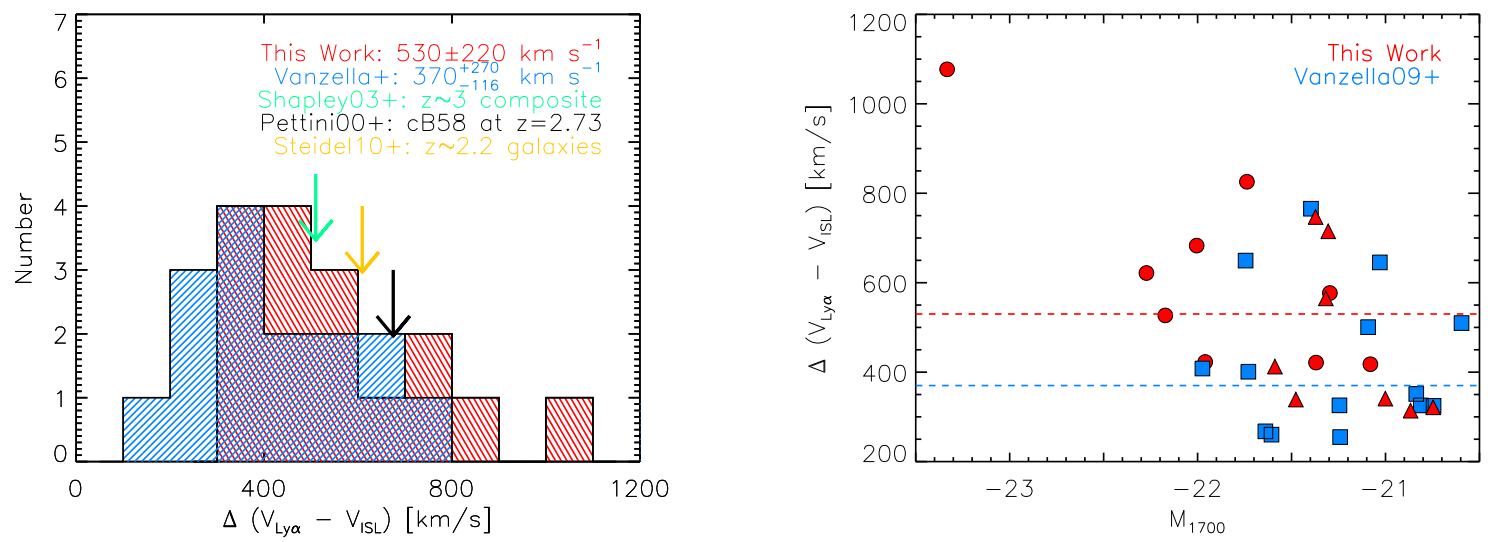

FIG. 5.- Left: The red histogram shows the distribution of the relative velocity between Ly $\alpha$ emission and interstellar absorption lines, measured from the 18 galaxies that clearly show both. The distribution of less UV-luminous $z \simeq 3.7$ galaxies in the GOODS South field measured by Vanzella et al. (2009) is shown in a blue hatched histogram. The median velocity of the galaxies in our sample is larger by $\approx 160 \mathrm{~km} \mathrm{~s}^{-1}$ than the Vanzella sample. The same quantities measured for a large sample of $z \simeq 3$ galaxies (Shapley et al. 2003) and that of MS 1512-cB58 (Pettini et al. 2000) are also indicated. Right: The correlation between the relative velocity inferred from the Ly $\alpha$ emission and interstellar absorption redshifts, $\Delta\left(v_{\mathrm{Ly} \alpha}-v_{\mathrm{abs}}\right)$, and UV luminosity $\left(M_{1700}\right)$ is shown together with the Vanzella sample (filled squares). The galaxies that are detected (undetected) in the IRAC data are shown in filled circles (triangles).

The outflow velocity is, by definition, the velocity of the ISM material relative to the galaxy's systemic redshift. The latter is usually measured by either stellar absorption lines or non-resonant emission lines from ionized or neutral gas. Unfortunately, most nebular emission lines in the rest-frame UV (e.g., Ly $\alpha$ and C IV; covered by our observed optical spectra) arise from resonant transitions and are strongly affected by radiative transfer effects; the ones that are not resonant (e.g., He II, C III]) are generally too weak to have significant detections in the existing spectra. In addition, at the redshift of our sample the most prominent nebular lines (such as $\mathrm{H} \alpha,[\mathrm{O}$ III $] 4959,5007,[\mathrm{O}$ II] 3727) are redshifted into the near-infrared (some beyond the $K$-band) at $z \gtrsim 3.8$.

Instead, we measure the relative velocity between the Ly $\alpha$ emission and that of the interstellar lines as a proxy for the outflowing medium. Verhamme et al. (2006) showed that the emergent Ly $\alpha$ profile observed at high redshift can be qualitatively reproduced by a simple model in which the energy from supernovae explosions drives an expanding shell of uniform velocity $V_{\text {exp. In }}$ their model, the Ly $\alpha$ photons that are back-scattered from the receding end of the expanding shell have a higher escape fraction by being scattered out of the core of the line; this results in redshifting the peak of the Ly $\alpha$ emission by a relative velocity of $\delta v=2 V_{\text {exp. }}$. On the other hand, interstellar absorption in the advancing side (i.e., the observer's side) of the expanding medium causes a trough that is blueshifted by $\delta v=-V_{\exp }$. In reality, there is observational evidence that some of the details of the Ly $\alpha$ profile is inconsistent with such a simple model Quider et al. 2009; Kulas et al. 2012) not to mention that there is evidence that ISM is neither uniform nor at a constant velocity (e.g., Steidel et al. 2010). Nevertheless, if both Ly $\alpha$ emission and interstellar absorption are primarily shaped by the outflowing medium, the relative velocity between the two should roughly trace the outflow velocity to first order.

We measured the relative velocity $\Delta V \equiv V_{\mathrm{Ly} \alpha}-V_{\mathrm{ISL}}$ between the interstellar and $\operatorname{Ly} \alpha$ features for the 17 galaxies with spectra of sufficient $\mathrm{S} / \mathrm{N}$ to detect both.
The velocity ranges from $235 \mathrm{~km} \mathrm{~s}^{-1}$ to $1140 \mathrm{~km} \mathrm{~s}^{-1}$ as presented in Figure 5. The median (mean) value for the sample is 527 (531) $\mathrm{km} \mathrm{s}^{-1}$ with the standard deviation of $220 \mathrm{~km} \mathrm{~s}^{-1}$. These values are significantly larger than the offset expected due to the intergalactic absorption alone ${ }^{8}$, and thus strongly supports the presence of the ISM in motion. In Figure [5 (left), we show the relative velocity distribution of the 17 galaxies in our sample, along with the measurements for the highly magnified $z=2.96$ galaxy MS 1512-cB58 and the average values for $z \simeq 3$ and $\simeq 2$ star-forming galaxies (Pettini et al. 2000; Shapley et al. 2003; Steidel et al. 2010, respectively). Also shown is the velocity distribution of 16 galaxies at $z \simeq 3.7$ measured by Vanzella et al. (2009). The median velocity measured for the Vanzella sample is $370 \mathrm{~km} \mathrm{~s}^{-1}, \approx 160 \mathrm{~km} \mathrm{~s}^{-1}$ lower than the value for our sample, although both have a substantial scatter. To test the significance of the difference between the two distributions, we used the Kolmogorov-Smirnov test (K$\mathrm{S}$ test, hereafter) in IDL kstwo.pro. The test returned the probability $P=0.286$ that the two measurements are drawn randomly from the same distribution.

Using all 33 galaxies (from our study and the Vanzella et al. 2009 sample) with $\Delta V$ measurements, we investigate whether the outflow velocity varies with UV luminosity and stellar mass. Figure 5 (right) shows the outflow measure $\Delta V$ as a function of UV luminosity, $M_{1700}$. The two horizontal lines mark the median value for the two samples. The data show a lot of scatter, as might be expected if orientation geometry, radiative transfer effects, or other physical processes play a role in determining the average $\Delta V$ of a galaxy. However, there is a suggestion that the "upper envelope" of the outflow velocity is larger for more luminous galaxies. We used two statistics to test for the presence of a correlation: Kendall's $\tau$ and Spearman $\rho$ tests. Both methods measure the test statistics $\left(\rho_{\mathrm{SR}}\right.$ or $\left.\tau_{\mathrm{K}}\right)$ and the probability of a null hy-

8 Assuming the Madau (1995) prescription and an initially Gaussian line profile, the IGM absorption causes the centroid of Ly $\alpha$ line to shift by $-70 \mathrm{~km} \mathrm{~s}^{-1}$ at the median redshift of $z=3.7$. The shift ranges over $-(50-85) \mathrm{km} \mathrm{s}^{-1}$ at the redshift range of $z=3.3-4.1$. 
pothesis (i.e., no correlation; $P_{\mathrm{SR}}$ or $\left.P_{\mathrm{K}}\right)$. The tests on our sample of 17 galaxies returned $(-0.593,0.012)$ and $(-0.456,0.011)$ for the Spearman $\rho$ and Kendall $\tau$ test, respectively, and reject the null hypothesis (no correlation) at the $\approx 99 \%$ level. Inclusion of the Vanzella sample results in measures of $(-0.289,0.103)$ and $(-0.216,0.077)$, slightly weakening the strength of this statement, but still suggestive of a weak correlation between our outflow velocity measure $\Delta V$ and the UV luminosity of a galaxy. Because the most luminous galaxy in our sample (BD18449) also has the highest velocity offset (1140 $\mathrm{km} \mathrm{s}{ }^{-1}$ ), we repeated the same tests excluding the object. The probability of null hypothesis is $4 \%(P=0.04)$ for our sample (16 galaxies), and $17 \%$ for the combined sample (32 galaxies).

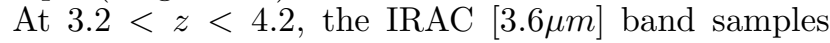
the rest-frame $I$-band, and thus can be used as a proxy for the stellar mass content. Unfortunately, the existing data from the Spitzer Deep Wide-Field Survey (SDWFS; Ashby et al. 2009) are too shallow to robustly detect $z \simeq 3.7$ galaxies with high $\mathrm{S} / \mathrm{N}$ (the $3 \sigma$ depth at $3.6 \mu \mathrm{m}$ is $23.29 \mathrm{AB}$ mag; see Table 5 of Ashby et al. 2009) . Of the 17 galaxies with outflow measurements, 6 are detected in the $[3.6 \mu \mathrm{m}]$ SDWFS imaging, 3 are ambiguous, and the remaining 8 are not detected. In Figure 5 (right), the IRAC-detected and IRAC-undetected sources are marked as red circles and triangles, respectively. The Vanzella sample is drawn from the GOODSSouth survey field which has much deeper Spitzer coverage (the $3 \sigma$ depth at $3.6 \mu \mathrm{m}$ of $26.75 \mathrm{AB}$; see Table 1 of Lee et al. 2012b). All 16 galaxies in the Vanzella sample are securely detected at $[3.6 \mu \mathrm{m}]$ with magnitudes ranging over $22.88-25.43 \mathrm{AB}$ mag with a median of 23.96 AB mag. Given the depth of the SDWFS data, only two of the galaxies in the Vanzella sample would have been detected had they been in the Boötes field.

Within our sample, there is weak evidence of a correlation between outflow velocity and IRAC brightness. The median (mean) relative velocities are $\Delta V \equiv\left(V_{\mathrm{Ly} \alpha}-\right.$ $\left.V_{\text {abs }}\right)=622(655) \pm 226$ and $412(481) \pm 213 \mathrm{~km} \mathrm{~s}^{-1}$ for the IRAC-detected and IRAC-undetected galaxies, respectively. Removing the outlying galaxy with the highest velocity, BD18449, from the IRAC-detected subset changes the median to $577(566) \pm 99 \mathrm{~km} \mathrm{~s}^{-1}$. If we include the two galaxies that are marginally detected in IRAC, the relative velocity decreases slightly to $577(593) \pm 219$ and $526(524) \pm 108 \mathrm{~km} \mathrm{~s}^{-1}$ with and without the source BD18449, respectively. In short, IRAC-bright galaxies appear to have a higher value of $\Delta V$ than their fainter counterparts. Because we do not have robust IRAC photometry for the majority of our galaxies, we are unable to directly test a bivariate correlation. A $\mathrm{K}-\mathrm{S}$ test indicates that the distribution of the IRAC-undetected galaxies in our sample is statistically identical to that of the Vanzella sample. On the other hand, the IRAC-detected galaxies are likely drawn from a different distribution, with just $7.9 \%$ probability of a null hypothesis when compared against the Vanzella sample.

When we consider only the galaxies within the Vanzella sample, we find no correlation with luminosity, IRAC brightness, or stellar mass. The test statistics are inconclusive, and can only rule out the null hypothesis at the $\approx 45 \%$ probability level.
It is not clear why our sample shows a correlation while the Vanzella sample does not. One possible explanation is that our sample probes a wider dynamic range of UV and optical rest-frame luminosities and thus is better suited to discerning a trend. The unknown viewing angle of our observations relative to the direction of the outflows will result in a large scatter in the observed velocities of outflows, which can wash away any trend if the dynamic range is not large enough. While better sampling of galaxies at the most luminous or massive end is clearly needed to place the constraints on a firmer footing, our results are qualitatively consistent with studies of lower-redshift star-forming galaxies (Martin 2005; Rupke et al. 2005; Weiner et al. 2009), where the outflow velocity increases with a galaxy's SFR and mass.

\subsection{The Average Physical Properties of the Luminous $z \simeq 3.7$ Galaxies}

Creating a high $\mathrm{S} / \mathrm{N}$ composite spectrum, by averaging over many individual spectra, can allow us to discern spectral features that are not typically visible in low-S/N individual spectra, and thereby infer the physical properties of the "typical" sample galaxy in a greater detail (e.g., Steidel et al. 2001; Shapley et al. 2003; Vanzella et al. 2009). The main challenge of constructing a composite spectrum is the fact that we do not have a good estimate of the systemic velocity of each galaxy. The spectral features we use for redshift identification, Ly $\alpha$ emission and/or interstellar absorption lines, are biased with respect to the systemic velocity. As discussed in 4.2, the interstellar absorption lines are often observed to be blue shifted with respect to the galaxy's systemic redshift, whereas the peak of the Ly $\alpha$ emission is redshifted due to radiative transfer effects.

Deriving the systemic redshift using a statistical correction is difficult to justify. Most derived corrections are based on LBG samples at lower luminosity and / or lower redshift (e.g., Adelberger et al. 2003; Steidel et al. 2010). In light of our findings in 4.2 that the outflow velocity may depend on the galaxy's luminosity, the suggested values from those studies may not reasonably apply to our sample. Instead, we compute a fiducial redshift for each galaxy and use it to de-redshift each spectrum. For the galaxies with only interstellar absorption lines, which we refer to as "abs"-type galaxies, the fiducial redshift is computed by applying a constant offset $v$ to the measured interstellar redshift; i.e., $z_{\text {fid }}=\left(-v / c+z_{\mathrm{IS}}\right) /(1+v / c)$ where $c$ is the speed of light. We assumed the offset value of $v=-250 \mathrm{~km} \mathrm{~s}^{-1}$, which places $\mathrm{S} \mathrm{V}$ photospheric absorption line at the correct vacuum wavelength, i.e., $\lambda=1501.76 \AA$. As for the galaxies with both $\operatorname{Ly} \alpha$ and interstellar redshifts ("comp"-type), we take the mean of the two as fiducial redshift. This approach should minimize the effect from the range of outflow velocities observed in our sample on our composite spectrum, by making a larger correction if the outflow velocity of a given galaxy is larger. Furthermore, the mean correction applied to the "comp" galaxies should be $v \simeq-250 \mathrm{~km} \mathrm{~s}^{-1}$ with respect to the interstellar redshifts (see Figure 5. the median velocity offset between Ly $\alpha$ and IS components is $530 \mathrm{~km} \mathrm{~s}^{-1}$ ), similar to that applied to the "abs" galaxies.

Because we base our stacking on the interstellar redshifts, we therefore limit our analyses only to the galaxies 
with measured interstellar redshifts. All but four galaxies in our sample have reliable interstellar redshifts determined from at least two absorption lines, while only half (20) have detectable Ly $\alpha$ emission. Another advantage of limiting the analyses to those with interstellar redshifts is that the composite spectrum created as a result should provide a fair representation of a flux-limited sample of high-redshift galaxies with no bias towards the Ly $\alpha$ line-emitting galaxies.

We created the composite spectra as follows. First, we determine the fiducial redshift for each galaxy as described above. Second, we resample each spectrum to a common (near) rest-frame wavelength vector using cubic spline interpolation (IDL interpol.pro with spline option). Third, we normalize each spectrum using the average flux density at $1430-1470 \AA$, where the spectrum is devoid of strong interstellar lines. Fourth, at each resampled wavelength element, we average the individual spectra weighting by the inverse variance (where the inverse variance is derived from the $1 \sigma$ error spectrum provided by the reduction pipeline; see Cooper et al. 2012). For each individual spectrum, the regions contaminated by atmospheric $A$ and $B$ band absorption (at $\lambda=7592-7675 \AA$ and $6886-6881 \AA$, respectively) are masked and excluded from the average. Since the error spectrum is background-limited, each galaxy has roughly equal weight in the average regardless of its luminosity.

In Figure 6. we show the composite spectrum constructed from 36 galaxies in our sample; i.e., all excluding five galaxies with no interstellar redshift. In the spectrum, we detect $\mathrm{C}$ III $\lambda 1176$ line and $\mathrm{S}$ V $\lambda 1502$, which arise from stellar photospheric absorption. The lines are at the correct wavelengths, therefore confirming that our final spectrum is in fact at systemic. Finally, we note that the bump in the error spectrum at $\lambda_{\text {rest }} \approx 1630 \AA$ shown in Figure 6 (also in Figure 7) is a result of the atmospheric $A$ band entering into the region for many galaxies in our sample, and is not related to the He II emission.

\subsubsection{The Galaxies with no Lyo emission}

In Figure 7, we show the composite spectrum for our $z \simeq 3.7$ sample constructed from 20 sources with interstellar absorption line redshifts and no Ly $\alpha$ emission. The spectrum clearly shows not only strong interstellar lines such as Si II $\lambda 1260$, O I+Si II $\lambda 1303$, C II $\lambda 1334$, Si IV $\lambda \lambda 1393,1402$, Si II $\lambda 1526$, C IV $\lambda \lambda 1548,1550$, Fe II $\lambda 1608$, and Al II $\lambda 1670$, but also weaker features such as Ni II $\lambda 1370$, Ly $\beta$, Si III $\lambda 1206$, N v $\lambda 1239,1243$.

The top panel of Figure 7 compares our composite spectrum with that of the average $z \simeq 3$ LBG from Shapley et al. (2003). Specifically, the Shapley et al. (2003) spectrum shown is the composite constructed by averaging the 199 galaxies in the bottom quartile of the Ly $\alpha$ EW distribution (i.e., referred to as "Group 1" by Shapley et al.; see their Table 3) and exhibits the strongest interstellar absorption. At $\lambda_{\text {rest }}>1216 \AA$, the $z \simeq 3$ and $z \simeq 3.7$ composite spectra closely mirror each other, even down to the small scale wiggles which likely result from weak nebular/interstellar features. The Ly $\alpha$ line morphologies blue-ward of the trough are also similar in the two spectra, suggesting that the column density and velocity distribution of the absorbing gas are com- parable. While the interstellar line ratios in our sample are generally similar to those of $z \simeq 3$ galaxies, the EWs appear to be slightly larger in our spectrum (see 4.5 later).

Despite the general similarities of our $z \simeq 3.7$ sample to the $z \simeq 3$ galaxies, there are also several features that may be distinct. Most notably, The C IV P Cygni emission, which traces stellar winds from massive stars and therefore sensitive to the stellar initial mass function and/or population ages (see Pettini et al. 2000, and references therein), may be enhanced for the $z \simeq 3.7 \mathrm{lu}-$ minous LBGs. Both spectra (top panel; Figure 7) are normalized at $1450 \AA$ and the continuum level at $1550 \AA$ immediately following the $\mathrm{C}$ IV emission is not well determined as can be seen in Figure 7. However, adjusting the normalization to a higher value would make the $\mathrm{P}$ Cygni emission more dramatically different. Although the difference is not statistically significant (as can be seen from the error spectrum on bottom of Figure 7), the composite spectra of lower-luminosity galaxies at $z \simeq 3.7$ presented in Vanzella et al. (2009, third row) and Jones et al. (2012, fourth row in Figure 7) also appear to exhibit similarly strong C IV emission, lending support to the possibility that $\mathrm{C}$ IV emission may have been indeed stronger at higher redshift.

We also detect N v $\lambda 1240$ P Cygni feature at a lower significance. The absorption of the $\mathrm{N}$ V doublet is not seen in the $z \simeq 3$ Shapley composite, but observed in the UV spectrum of MS1512-cB58 to have $W_{0}=0.23 \AA$ (second panel of Figure 7 Pettini et al. 2002). In fact, the line morphologies of both $\mathrm{C}$ IV and $\mathrm{N} V$ in our spectrum are more consistent with that of cB58 than with the $z \simeq 3$ composite, even though cB58 is observed with a much higher covering fraction (the residual intensity in the line cores is nearly zero). Like $\mathrm{C} \mathrm{IV}, \mathrm{N}$ V also traces stellar winds from massive $\mathrm{O}$ stars and is thus also sensitive to the galaxy's IMF, age, and metallicity.

We also detect possible weak absorption lines of Ni II $\lambda 1370$, and Ni II $\lambda 1317$, which are absent in the Shapley et al. (2003) spectrum but are detected in the UV spectrum of MS1512-cB58 at a $W_{0} \simeq 0.2 \AA$ level (Pettini et al. 2002). Ni is one of the Fe-peak elements released by Type Ia supernovae $(\mathrm{SNe})$ and is found to be underabundant in the cB58 spectrum by a factor of three relative to, e.g., Si. Pettini et al. (2002) argue that the observed underabundance of the Fe-peak elements in cB58 is evidence of a young ( 300 Myr) population age, since metal enrichment by longer-lived, intermediate-mass stars has not yet caught up with the elements released by short-lived OB stars. The tentative detection of $\mathrm{Ni}$ in our luminous $z \simeq 3.7$ galaxies may suggest that these are "older" (i.e., have larger UV-luminosity weighted ages) than the lower-luminosity $z \simeq 3$ galaxies, perhaps because the more luminous galaxies have a more extended star-forming phase. Interestingly, population ages inferred from photometric measurements of the Balmer break strength do no vary significantly with UV luminosities (e.g., Lee et al. 2011; Oesch et al. 2012), thus suggesting otherwise. However, such measurements are almost always made on photometric samples, and typically show large scatter and uncertainty. While the current data have insufficient $\mathrm{S} / \mathrm{N}$ to infer galaxy ages from absorption lines properties, fu- 


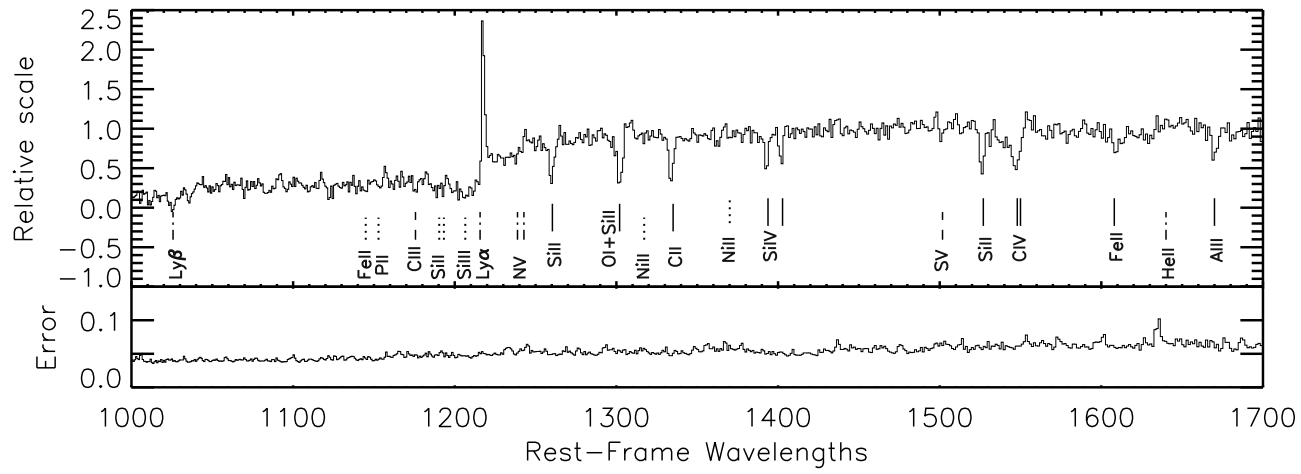

FIG. 6. - The average spectrum of all 36 confirmed star-forming galaxies at $3.2<z<4.6$ together with the error spectrum on bottom. The spectrum is normalized at $1430-1470 \AA$. Also marked are the vacuum wavelengths of prominent stellar photospheric (dashed), nebular (dashed-dot), and interstellar lines (strong lines in solid, weaker lines in dotted lines, respectively). Note that while the expected line centroids are labeled, not all these features are detected in the composite spectrum.

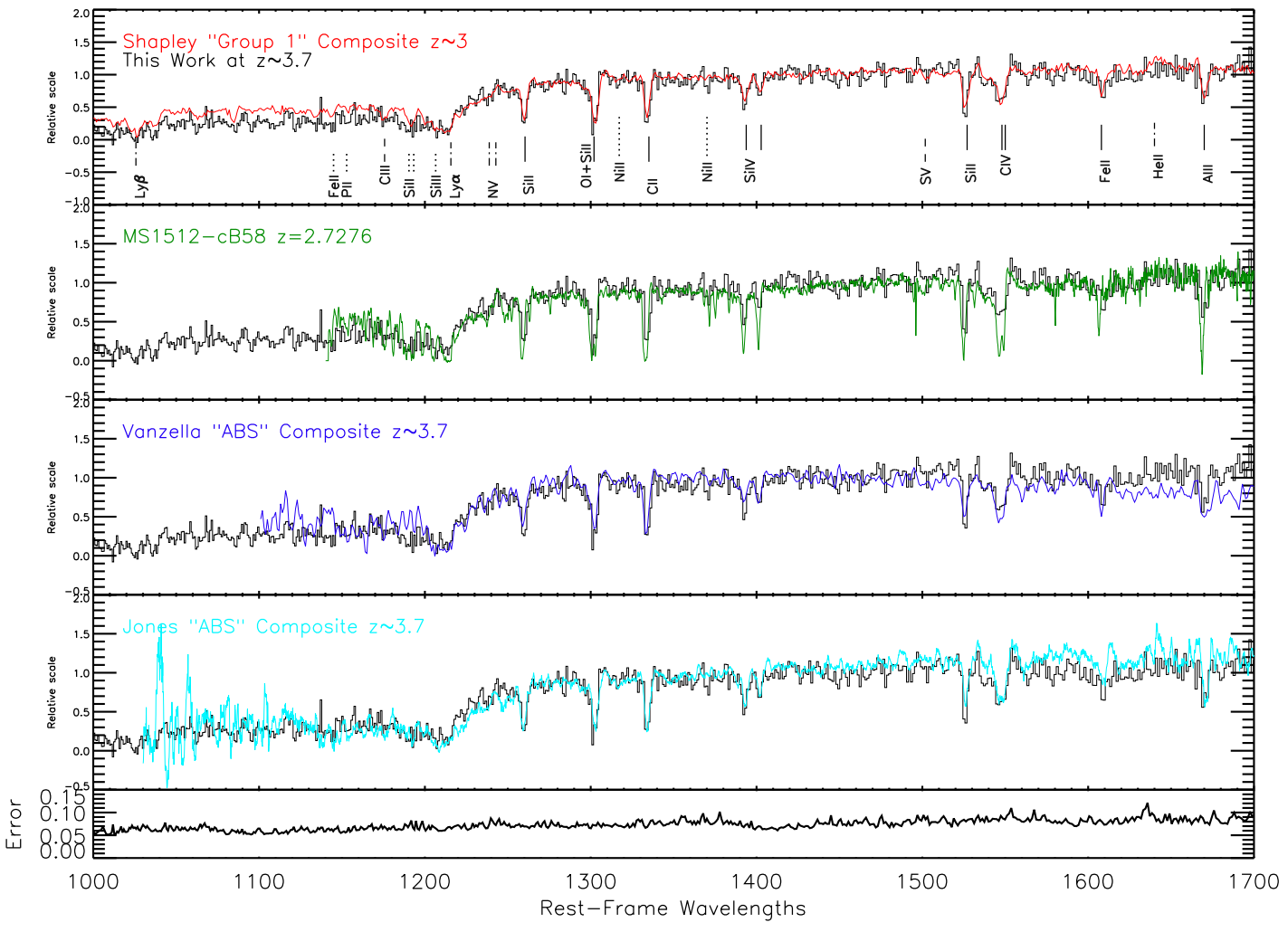

FIG. 7. - The top panel shows average spectrum of galaxies without Ly $\alpha$ emission in our sample (black)compared with the composite spectrum of the $z \simeq 3$ galaxies from Shapley et al. (2003) (red). The Shapley et al. spectrum shown here is their "Group 1" composite which averages spectra of galaxies with Ly $\alpha$ EW in the bottom quartile of the overall distribution (see Shaplev et al. 2003, for details). The lower panels show the same average spectrum of our sample compared with the spectra of MS1512-cB58 (second panel Pettini et al. 2002); the $z \simeq 3.7$ composite of lower-luminosity galaxies (third and fourth panel, see Vanzella et al. 2009; Jones et al.|2012, respectively). Both composites are created from galaxies with no Ly $\alpha$ emission.

ture observations of deep, high-resolution spectroscopy of UV-luminous galaxies should provide independent estimates on galaxy's population age (as well as metallicity, and IMF).

We compare our composite spectrum with the Vanzella et al. (2009) "abs"-class composite created from 21 less-luminous $z \simeq 3.7$ galaxies which lack Ly $\alpha$ emission (third panel of Figure 7). The absorption features are clearly stronger in our spectrum in most cases. The difference appears to be mainly luminosity-dependent as both composites have similar Ly $\alpha$ absorption EW.
Our $z \simeq 3.7$ composite also has a redder UV slope than the Vanzella composite, consistent with the wellknown correlation between luminosity and UV colors (e.g., Bouwens et al. 2009; Finkelstein et al. 2012). As in the case of the Shapley composite spectrum, $\mathrm{Ni}$ is not detected in the Vanzella spectrum.

Finally, the bottom panel shows the "abs"-type galaxy composite from the data presented in Jones et al. (2012). The median luminosity of 24 galaxies included in the spectrum is $M_{\mathrm{UV}} \sim-21$. Their spectrum has a somewhat redder slope compared to our composite and the 


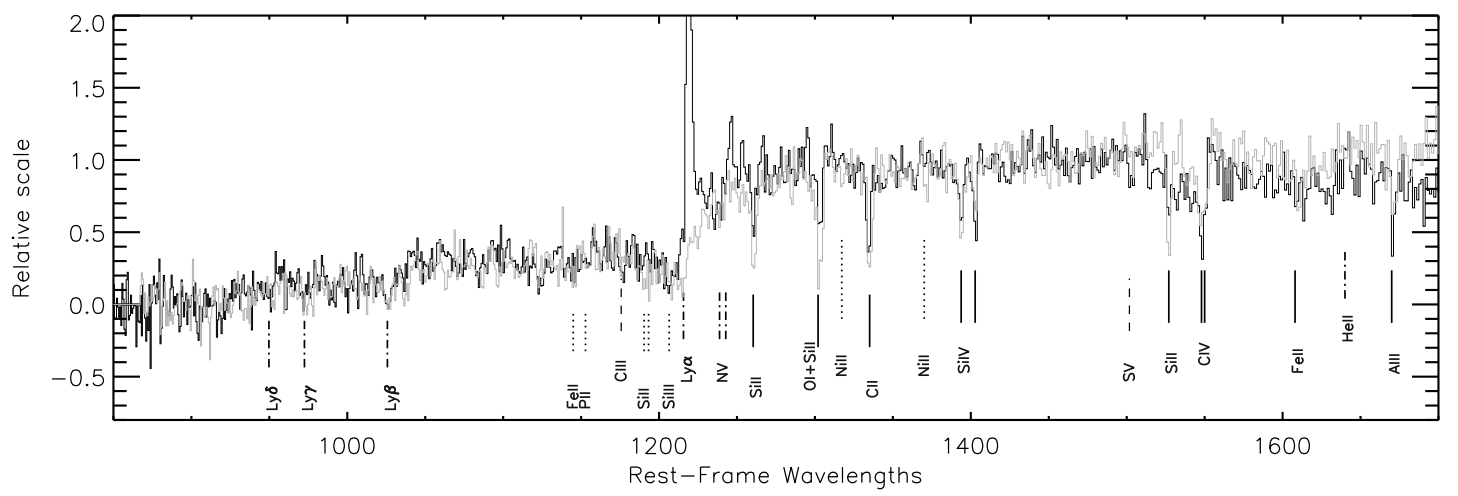

FIG. 8.- The composite spectra of the Ly $\alpha$-emitting galaxies (black) vs non-emitters (light grey) are shown. Both spectra are normalized at $1450 \AA$. Interstellar absorption is generally stronger in the non-emitter spectrum, consistent with the expectation of a higher gas column density in the ISM for the non-emitters. We also detect Ni II in the non-emitter spectrum but not in the emitter spectrum. Conversely, $\mathrm{S} \mathrm{V}$ is visible in the emitter spectrum, but not in the non-emitter spectrum (see discussions in 4.3 .2 ).

Vanzella composite, which may be due to systemic error in the flux calibration (T. Jones, private communication). The most notable differences are that the Jones et al. spectrum shows higher EWs in $\mathrm{Si}^{*}$ II emission (at $\lambda=1265,1309,1533 \AA$, but most pronounced at $1533 \AA$ ) and He II emission at $1640 \AA$. We discuss this in further detail in 4.4. Similar to the Vanzella composite, the interstellar absorption lines in the Jones composite are generally weaker compared to our spectrum. We compare the interstellar absorption lines from these spectra in further detail in 4.5 .

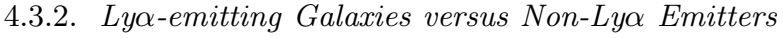

The origin of Ly $\alpha$ emission in high-redshift galaxies is still not well understood. The observed Ly $\alpha$ EW is a sensitive function of the galaxy's age, metallicity, and the amount and relative geometry of gas and dust in the ISM. In theory, comparing the physical properties of galaxies with or without Ly $\alpha$ emission can provide useful information about which parameter is the driving factor of the observed $\operatorname{Ly} \alpha$ emission in galaxies. Recent studies suggest that Ly $\alpha$-emitting galaxies are less dusty (Gawiser et al. 2006; Pentericci et al. 2007; Kornei et al. 2010) on average than the non-emitters, although it is possible for very dusty galaxies to exhibit strong Ly $\alpha$ emission (e.g., Finkelstein et al. 2009, 2011, and the references therein). Furthermore, different studies have conflicting results about how the Ly $\alpha$ emission is related to the galaxy's population age (Pentericci et al. 2009; Kornei et al. 2010).

One complication that has hindered obtaining a clear picture has been that the galaxies observed with $\mathrm{Ly} \alpha$ emission tend to be intrinsically less-luminous in their continuum emission. Combined with the spectroscopic selection effect (i.e., that it is easier to spectroscopically confirm galaxies with strong $\operatorname{Ly} \alpha$ emission than those without), it is challenging to compare fairly the physical properties of the emitter and non-emitter populations.

We directly compare the spectroscopic properties of the galaxies observed with and without Ly $\alpha$ emission by stacking them separately. Once again, we only consider the 36 (of the 41) galaxies with the measured interstellar redshifts. Of these, 17 galaxies exhibit Ly $\alpha$ emission and 20 do not. One galaxy in the Ly $\alpha$-emitting subsample has unusually strong $\mathrm{N}$ V emission, and we exclude this from the stack. We stacked the remaining 16 Ly $\alpha$-emitters and 20 non-emitters as described previously. The spectrum of non-emitters is identical to that shown in Figure 7.

Figure 8 compares the two composite spectra (normalized at $1450 \AA$ ). The median (mean) UV luminosities of the two samples are comparable: $M_{1700}$ is $-21.37(-21.56)$ and $-21.52(-21.62)$ for the emitter and non-emitter subsamples, respectively. Similarly, the median (mean) Iband magnitudes are also comparable: 24.58 (24.40) and 24.41 (24.28), respectively.

It is clear from the spectra that most of the strongest interstellar absorption lines, including Si II, O I+Si II, $\mathrm{C}$ II and Si IV $\lambda 1394$, are stronger in the non-emitter composite spectrum. The anti-correlation between the EWs of Ly $\alpha$ emission and interstellar absorption was also observed in the $z \simeq 3$ galaxies by Shapley et al. (2003). Shapley et al. argued it as evidence that the emergent Ly $\alpha$ strength and interstellar absorption features are jointly determined by random sight lines seen through the regions of differing optical depths: more optically thick sightlines result in more absorption/attenuation of Ly $\alpha$ and stronger interstellar absorption.

We also observe several subtle but intriguing features that have not been previously reported. The two Ni II lines detected in the non-emitter spectrum are absent in the emitter spectrum. Although both Ni lines are detected at a low $\mathrm{S} / \mathrm{N}$ level, the fact that both appear in one spectrum and neither does in the other suggests that the trend is in fact real. In addition, S v line is clearly detected in the emitter spectrum but not in the non-emitter spectrum. Ni is one of the Fe-peak elements (Ni, Fe, Mn) that are released by Type Ia SNe, while Type II SNe are the main producers of $\mathrm{S}$ (together with $\mathrm{Si}, \mathrm{O}, \mathrm{Mg}, \mathrm{P}$ ). Another Type II element, $\mathrm{N}$ v, is visible in the non-emitter population only. In the context of standard chemical evolution models, these observations provide unique insights into the recent star-formation history of the dominant stellar population. Based on these findings, we speculate that the luminous non-emitter population may have continued star-formation for a considerably longer period of time than the Ly $\alpha$-emitting population, as the latter have not had enough time to return metals produced by intermediate-mass, longer-lived stars into the ISM (Pettini et al. 2002). S V is not detected in either 
Jones et al. (2012) or Vanzella et al. (2009) emitter spectrum, suggesting that the process responsible for this line may also depend on luminosity (or, more likely, age).

\subsection{Emission Line Properties}

We detect several weak $\mathrm{Si}$ emission line features in the composite spectrum. Si II $^{*}$ emission lines $(\lambda=$ 1265,1309 , and $1533 \AA$ ) are thought to arise from the fine-structure transitions within the outflowing gas in the ISMand CGM (Shapley et al. 2003). Photons absorbed by a ground-state Si II ion is re-emitted either at the same energy level or at the corresponding finestructure transition with roughly equal probabilities. In Figure 9, we compare our composite spectrum (shown in Figure 6) with those presented in Shapley et al. (2003) and Jones et al. (2012) at $z \simeq 3$ and $\simeq 4$, respectively. The $\mathrm{Si} \mathrm{II}^{*}$ emission profiles in our spectrum are similar to those in the Shapley spectrum, while the Jones et al. (2012) spectrum shows clear excess Si II* emission, most pronounced at $1533 \AA$.

In the case of optically thick gas, resonant Si II photons will be continuously scattered until they emerge as Si II*, and therefore, the net EW of the Si II transitions should be zero; i.e., $W_{\text {SiII }}$.em $+W_{\text {SiII,abs }}=0$. Contrary to this expectation, Jones et al. (2012) reported that the measured value for their $z \simeq 4$ sample is

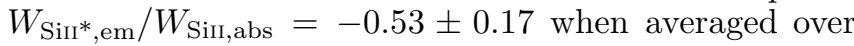
the two strongest Si II transitions at 1260 and $1527 \AA$. In comparison, the analogous value measured from the Shapley composite at $z \simeq 3$ is $-0.16 \pm 0.04$. Jones et al. (2012) argued that the lower-than-expected strength of fine-structure emission is likely due to the fact that much of the emission arises in the CGM and therefore is not sampled within their $1^{\prime \prime}$ slitlets $(\sim 7 \mathrm{kpc})$. They further argued that the radius of fine-structure emission may increase with redshift, thus giving rise to a higher line ratio $W_{\text {SiII*,em }} / W_{\text {SiII,abs }}$ at higher redshift as mentioned above. However, our DEIMOS observations cast doubt on this interpretation. Even though we used larger slit widths $\left(1.2^{\prime \prime}\right.$ sampling $8.6 \mathrm{kpc}$ at $\left.z=3.7\right)$ than the Jones et al observations, the emission level is still comparable to that of the Shapley spectrum. One possible explanation is that the size of the $\mathrm{Si} \mathrm{II}^{*}$-emitting CGM region is a strongly increasing function of galaxy luminosity rather than of redshift as Jones et al. (2012) proposed.

Another notable emission line in the rest-frame ultraviolet spectrum is He II emission, which is clearly detected in the Shapley et al. (2003) and Jones et al. (2012) samples. The line profile of the He II emission (FWHM $\sim 1500 \mathrm{~km} \mathrm{~s}^{-1}$ at $z \simeq 3$ ) suggests that the emission has been broadened significantly by fast, dense winds from Wolf-Rayet (W-R) stars (Shapley et al. 2003). W-R stars, as descendants of O stars with masses $\gtrsim 20-30 M_{\odot}$, are short-lived, and thus the He II emission of W-R origin is strong only in the initial phase of star-formation (Schaerer \& Vacca 1998). Assuming constant star-formation history and solar metallicity, Schaerer \& Vacca (1998) estimated that EW(He II1640) should drop to zero within $\approx 20$ Myr. Assuming subsolar metallicity $\left(0.4 Z_{\odot}\right)$, the He II-bright phase decreases to just a few Myr.

Our data do not show a clear sign of He II emission (bottom right of Figure 9, also see Table 3) even though our spectrum is in reasonable agreement with the Shapley composite within (large) errors. We speculate that the lack of strong He II emission may be due to the fact that the galaxies in our sample may have more extended star-formation histories than those in the Shapley and Jones sample. While a higher-resolution, higher $\mathrm{S} / \mathrm{N}$ spectrum is necessary to place more robust constraints on the strength of the He II emission in the luminous galaxies, such an interpretation is qualitatively in line with the inferences from other observations discussed earlier (4.3.1 and 4.3.2) that the luminosity-weighted ages increase with UV luminosity and/or decrease with Ly $\alpha$ EW.

\subsection{Dependence of Interstellar Absorption Line Strengths on Galaxy Parameters}

The strength of interstellar absorption in the UV spectrum is primarily determined by the kinematics, ionic column density, and covering fraction of the absorbing gas in the ISM, and therefore provides direct probes of the physical conditions therein. The fact that the interstellar absorption EW correlates so strongly with Ly $\alpha$ EW suggests the same gas that produces absorption features is also responsible for attenuating the Ly $\alpha$ emission (e.g., Shapley et al. 2003; Vanzella et al. 2009). When the line is saturated, which may be the case for many of the strongest interstellar absorption features at high redshift, the EWs primarily depend on the velocity dispersion of the gas (measured by deconvolved full-widthat-half-maximum) and covering fraction $\left(C_{f}\right)$, and to a lesser degree, on the ionic column density. ${ }^{9}$ Hence, the measurements of such a correlation at different galaxy properties (e.g., luminosity, mass, color, morphology) and at different redshifts can provide valuable insights into the changing physical parameters in the ISM among the galaxies. Towards that end, we compare the measurements of interstellar lines with those in the literature. We mainly focus on the four strongest low-ionization interstellar (LIS) lines, Si II $\lambda 1260$, O I+Si II $\lambda 1303$, $\mathrm{C}$ II $\lambda 1334$, and $\mathrm{Si}$ II $\lambda 1527$, which trace cold neutral gas in the ISM. Following the convention used in Shapley et al. (2003) and Jones et al. (2012), we use the average of these four as the indicator of LIS line strength, and refer to it as $W_{\text {LIS }}$ hereafter.

We measured the $W_{\text {LIS }}$ from five distinct composite spectra: (1) the full sample (Figure 6), (2,3) Ly $\alpha$ emitting galaxies and non-emitters (Figure 8), and (4,5) two non-emitter samples binned by UV luminosity (divided at the median luminosity $M_{\mathrm{UV}}=-21.4$ ). We measured the line EWs directly from the composite spectra; we also independently measured the same lines from the composite spectra of the four subsamples presented in Shapley et al. (2003), and confirmed that our measurements return very similar values (within $0.1 \AA$ ) to their published values. These results are shown in Figure 10 and tabulated in Table 3. In the figure, the value for our full sample is shown by a large open circle, while the two subsamples binned by Ly $\alpha \mathrm{EW}$ are shown by

9 The equivalent width of an unsaturated absorption line should obey $W_{\text {IS }} \propto N_{\text {ion }}$; for a saturated line, $W_{\text {IS }} \propto b\left[\ln \left(N_{\text {ion }} / b\right)\right]^{0.5}$ while $f_{\max } / f_{\text {cont }}=1-C_{f}$ where $f_{\max }$ is the intrinsic flux density at maximum absorption, $b$ is Doppler parameter, and $C_{f}$ is covering fraction of the absorbing gas. 

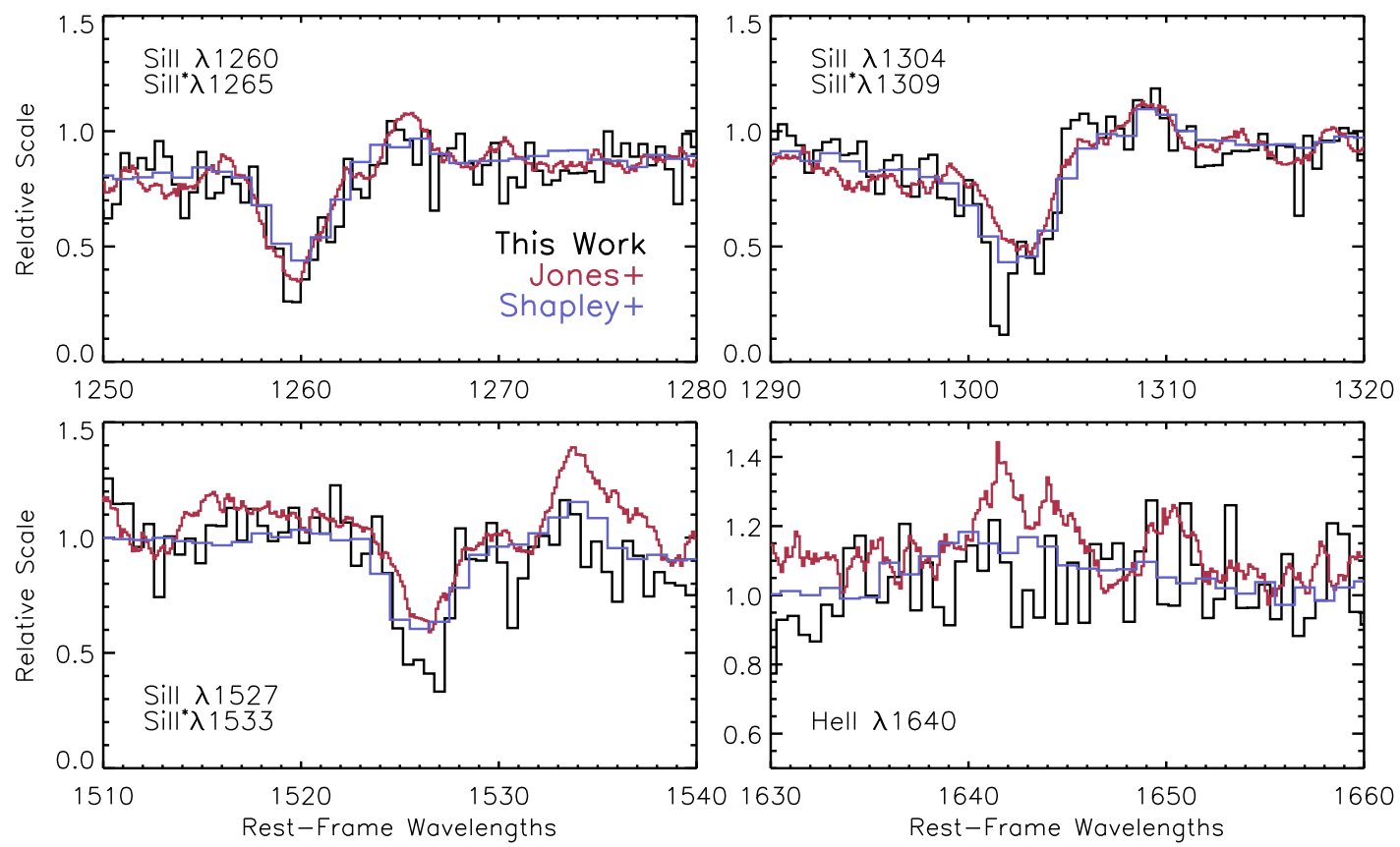

FIG. 9.- The composite spectrum showing the regions around Si II* emission lines and He II is compared with composite spectra of $z \simeq 3$ and $z \simeq 4$ LBGs from Shapley et al. (2003) and Jones et al. (2012) respectively. The Si II* emission line profiles are very similar to those of the $z \simeq 3$ Shapley et al. (2003) composite, while the Jones et al. (2012) spectrum shows more pronounced emission. We do not detect $\mathrm{He}$ II emission in our composite spectrum, although within margin of error ( $\simeq 15 \%$ of continuum level), our spectrum is consistent with the $z \simeq 3$ Shapley et al. spectrum.

TABLE 3

Equivalent Widths Measured from the Composite Spectrum

\begin{tabular}{ccccccc}
\hline \hline Ion & $\lambda_{\text {rest }^{\mathrm{a}}}(\AA)$ & $W_{0, \text { full }}(\AA)$ & $W_{0, \text { emitters }}(\AA)$ & $W_{0, \text { non-emitters }}(\AA)$ & $W_{0, M_{\mathrm{UV}}<-21.4}{ }^{\mathrm{b}}(\AA)$ & $W_{0, M_{\mathrm{UV}} \geq-21.4}{ }^{\mathrm{b}}(\AA)$ \\
\hline H I & 1215.67 & $4.5 \pm 0.2$ & $15.8 \pm 0.1$ & $-4.3 \pm 0.5$ & $-4.9 \pm 0.2$ & $-3.2 \pm 0.4$ \\
Si II & 1260.42 & $-1.8 \pm 0.2$ & $-1.4 \pm 0.2$ & $-2.3 \pm 0.3$ & $-3.0 \pm 1.0$ & $-2.0 \pm 0.5$ \\
O I+Si II & 1303.27 & $-2.7 \pm 0.2$ & $-1.9 \pm 0.2$ & $-3.4 \pm 0.5$ & $-3.0 \pm 1.0$ & $-3.1 \pm 0.6$ \\
C II & 1334.53 & $-2.3 \pm 0.2$ & $-1.6 \pm 0.2$ & $-2.7 \pm 0.3$ & $-2.0 \pm 0.4$ & $-2.2 \pm 0.4$ \\
Si IV & 1393.76 & $-1.4 \pm 0.1$ & $-0.9 \pm 0.1$ & $-1.8 \pm 0.2$ & $-1.9 \pm 0.4$ & $-1.9 \pm 0.3$ \\
Si IV & 1402.77 & $-1.0 \pm 0.1$ & $-1.2 \pm 0.1$ & $-0.9 \pm 0.1$ & $-0.7 \pm 0.3$ & $-1.1 \pm 0.2$ \\
Si II & 1526.71 & $-1.7 \pm 0.2$ & $-0.8 \pm 0.2$ & $-2.3 \pm 0.3$ & $-2.4 \pm 0.6$ & $-2.1 \pm 0.3$ \\
C IV & 1549.48 & $-3.1 \pm 0.3$ & $-2.8 \pm 0.3$ & $-3.3 \pm 0.3$ & $-3.4 \pm 0.6$ & $-3.1 \pm 0.4$ \\
He II & 1640.40 & $0.3 \pm 0.3$ & $-0.2 \pm 0.5$ & $0.1 \pm 0.5$ & $0.3 \pm 0.6$ & $-1.1 \pm 1.0$ \\
\hline
\end{tabular}

a The vacuum wavelengths

b The sample only includes the non-emitters in the sample (i.e., $W_{0, \text { Ly } \alpha}<0 \AA$ )

filled circles. The measurements for two luminosity (nonemitters) bins are shown by filled diamonds. We also show the Shapley et al. (2003) and Jones et al. (2012) points as downward and upward triangles, respectively.

It is clear that Ly $\alpha$-emitting galaxies have much weaker interstellar absorption (by more than $1 \AA$ ), thus confirming the earlier studies. However, it is also true that our points for non-emitters and for the full sample lie systemically lower (i.e., stronger absorption) by $\approx 2 \sigma$ than the other samples when Ly $\alpha \mathrm{EW}$ is fixed. As a guide, we mark the $1 \sigma$ range by interpolating the three Shapley subsamples (except for the highest $W_{0, \mathrm{Ly} \alpha}$ bin; Group 4). All three Jones et al. (2012) points are consistent with this relation within the errors. The fact that the measurements at $z \simeq 3$ and $z \simeq 4$ are very similar at comparable luminosities $\left(\left\langle M_{\mathrm{UV}}\right\rangle=-21\right.$ : Shapley et al. 2003; Jones et al. 2012) rules out that we are seeing redshift evolution of the absorbing gas. Rather, it may be that the observed discrepancy is a luminosity-dependent effect because the median luminosity of our sample is
-21.4 ; i.e., $45 \%$ more luminous). We attempted to test the luminosity-dependence by splitting the nonemitter sample into two bins according to UV luminosity (diamonds in Figure 10), but were unable to measure any significant difference due to the large uncertainties (each composite had only 10 galaxies). Jones et al. (2012) also observed a luminosity-dependent trend in their measurements, i.e., the LIS absorption was weaker for lower-luminosity galaxies when $W_{\mathrm{Ly} \alpha}$ is fixed. Similarly, they reported some level of variations in LIS absorption on other galaxy parameters such as UV spectral slope, half-light radius, UV luminosity, and stellar mass (see their Figure 9). The trend that they observed is in the same direction as our observations; that moreluminous/redder/larger galaxies lie below the fiducial relation while less-luminous/bluer/smaller counterparts lie above the same relation.

Despite relatively large uncertainties in the Jones measurements and ours, these results show a hint of changing physical conditions within the ISM that depend on 


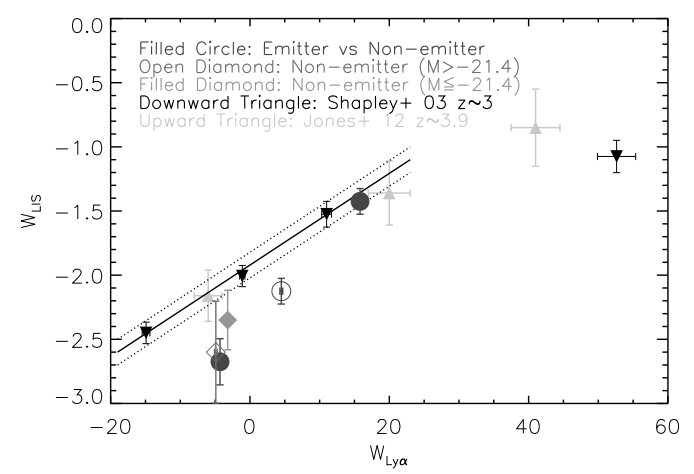

Fig. 10. - Equivalent width of low-ionization absorption lines as a function of Ly $\alpha$ EW. Large filled circles are from our sample divided into two bins as Ly $\alpha$ "emitters" and "non-emitters", while the large open circle shows the value measured for the full sample. Filled upward-pointing triangles show the measurements from the Jones et al. (2012) $z \simeq 3.9$ samples in three bins according to $\operatorname{Ly} \alpha \mathrm{EW}$, while downward-pointing triangles indicate similar measurements at $z \simeq 3$ as published in Shapley et al. (2003). Also shown are two luminosity bins $(M<-21.4$ and $M \geq-21.4)$ of non-emitters in filled and open diamond, respectively.

galaxy properties. Since many demographic properties of galaxies correlate well with one another (luminosity, color, size, stellar mass, total mass), it is not possible to pinpoint what may be the driving factor in producing the observed trends. Nevertheless, it is still worthwhile to speculate about possible physical scenarios that may give rise to the subtle differences observed. As mentioned above, our sample contains more luminous and therefore presumably more massive galaxies (e.g., Lee et al. 2006, 2011). We can therefore expect these galaxies to have higher velocity dispersions on average, thus contributing to increased EWs for the interstellar absorption lines. In addition, the covering fraction of the absorbing gas may be higher in more luminous galaxies. Such a scenario would not only imply deeper (more saturated) absorption lines in more luminous galaxies, but would also explain the observed decrease in the fraction of Ly $\alpha$-emitting galaxies towards higher luminosities (as discussed in 4.1 and Figure 4). There is also circumstantial evidence that more luminous galaxies are more reddened, suggesting a higher covering fraction of dust than in less luminous galaxies. If the dust and gas distributions are similar, this also supports an increased covering fraction of gas in more luminous galaxies. Finally, it may be possible that the more luminous galaxies have ISM that are more chemically enriched. However, this last speculation is difficult to confirm without much higher quality (and higher resolution) data.

\section{SPACE ODDITIES}

\subsection{A Protocluster Candidate at $z=3.78$}

The spectroscopic redshifts reveal the presence of a candidate large-scale structure at $z=3.78$. Five of the eleven galaxies on the mask BDm_02 are identified at redshifts $3.782<z_{\text {Ly } \alpha}<3.787$, within $1 \mathrm{Mpc}$ (physical) from one another. In Table 2, these galaxies are marked with superscript "g". In Figure 11, we show the relative positions of the eleven galaxies observed in the mask BDm_02 in redshift and angular distances, and also compare with the redshift distribution determined from the current compilation of spectroscopy of our photomet- ric candidates (see Lee et al. 2011, for further detail). As evident from the histogram, the redshift distribution on the mask BDm_02 is highly unusual in comparison with that of the overall population in our sample.

We determined interstellar redshifts for four of the five galaxies, with the values ranging over $z_{\mathrm{IS}}=3.773-3.779$. The difference between the interstellar and Ly $\alpha$ redshifts for these sources suggest the relative velocity of $310-530$ $\mathrm{km} \mathrm{s}^{-1}$. These values are comparable to the relative velocities determined for "field" galaxies, which we discussed in 4.2. Two galaxies (BD100006, BD100902) are individually detected in the SDWFS IRAC $[3.6 \mu \mathrm{m}]$ image, suggesting that they have larger stellar masses than the other three, assuming a similar star-formation history.

All five galaxies show pronounced Ly $\alpha$ emission as can be seen in the two-dimensional spectra (Figure 12). The mean and median EWs are $W_{0}=15.3 \AA$ and $14.2 \AA$, respectively, much higher than that determined for the full sample $W_{0}=-1.2 \AA$. Similar observations were made by Kuiper et al. (2012) for a $z=3.13$ structure near the radio galaxy MRC 0316-257. Three LBGs associated with the radio galaxy at $z=3.13$ have Ly $\alpha$ EWs of $W_{0}=14.9,39.3$, and $17.8 \AA$. Based on the effective EW measured from the stacked spectrum, Kuiper et al. (2012) estimated $W_{0}=26.4 \pm 3.8 \AA$ for the 3 protocluster galaxies compared with $7.4 \pm 2.1 \AA$ for 12 field galaxies. The observed high EW is unlikely to be influenced by the spectroscopic selection effect, as all three galaxies in the MRC 0316-257 overdensity and four (out of five) of the ones in our overdensity would have been identified via absorption line features even if the Ly $\alpha$ emission was not present. Hence, these observations suggest that LBGs in the most massive potential wells may have, on average, intrinsically stronger $\operatorname{Ly} \alpha$ emission than their counterparts in the field.

If the stronger Ly $\alpha$ emission observed for these galaxies is interpreted as higher SFRs, our results would imply that the galaxies in dense environments have, on average, higher SFRs than those in the field. This is the opposite of the SFR-density relation observed locally. The "reversal" of the SFR-density relation was already observed at $z \simeq 1$ (Elbaz et al. 2007; Cooper et al. 2008), where a large number of bright star-forming galaxies exist in group environments. Considering the fact that the SFRdensity relation is likely produced as a result of quenching that preferentially takes place in massive systems, the reversal of such a relation is not surprising at high redshift where most galaxies are still well below the quenching "threshold" in masses. Accurate measurements of their stellar masses and extinction properties (UV spectral slope as a proxy for reddening in UV, or direct measurements in the far-infrared) will provide the estimates of SFR and specific SFR thereby shedding more light on the nature of their Ly $\alpha$ emission.

Interestingly, the only two galaxies in our sample that exhibit doubly-peaked Ly $\alpha$ emission both belong to the $z=3.78$ structure (Figure 12. BD100902 and BD98176). While the frequency of similar sources at $z \simeq 2-3$ is only slightly lower 20 - 33\% (Kulas et al. 2012) than that observed in our sample, we speculate that it is possible that these galaxies may have intrinsically different physical parameters that affect the transmission of $\operatorname{Ly} \alpha$ photons 

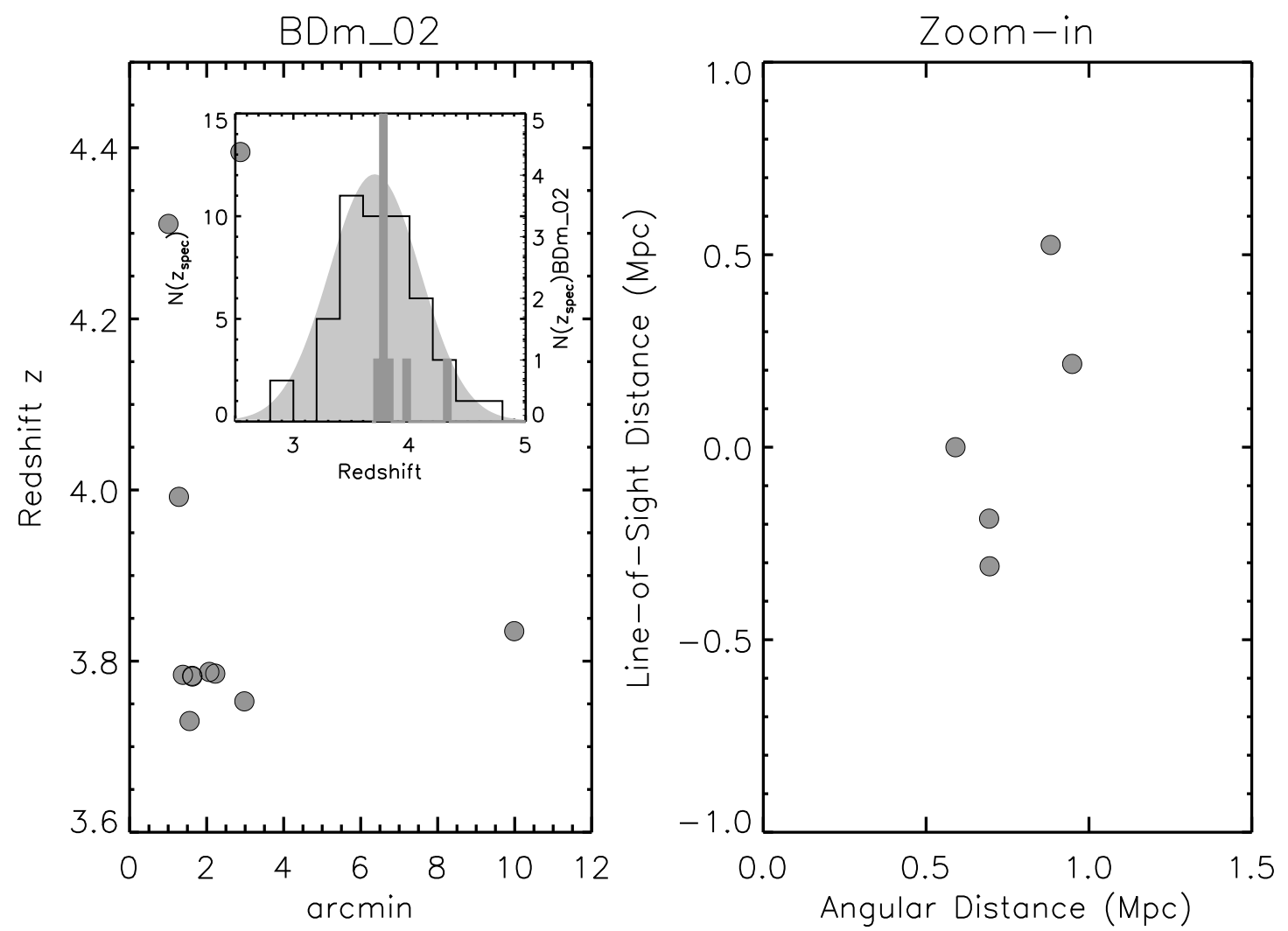

FIG. 11.- Left: The relative positions in angular and redshift space of 11 galaxies observed on the BDm_02 mask are shown in filled circles. The angular distance is computed from the center of the five galaxies; RA $=14^{\mathrm{h}} 31^{\mathrm{m}} 44.88^{\mathrm{s}}, \mathrm{DEC}=32^{\circ} 24^{\prime} 30.24^{\prime \prime}(\mathrm{J} 2000)$. In the inset, we show in open histogram the redshift distribution determined from the current spectroscopic sample compiled to date. The light grey shade illustrates the normal distribution with $\sigma_{z}=0.4$ centered at $z=3.7$. The redshift distribution of the galaxies on the BDm_02 is shown in dark grey histogram. Right: The zoom-in on the overdensity region. The angular positions are now shown in units of Mpc (physical).

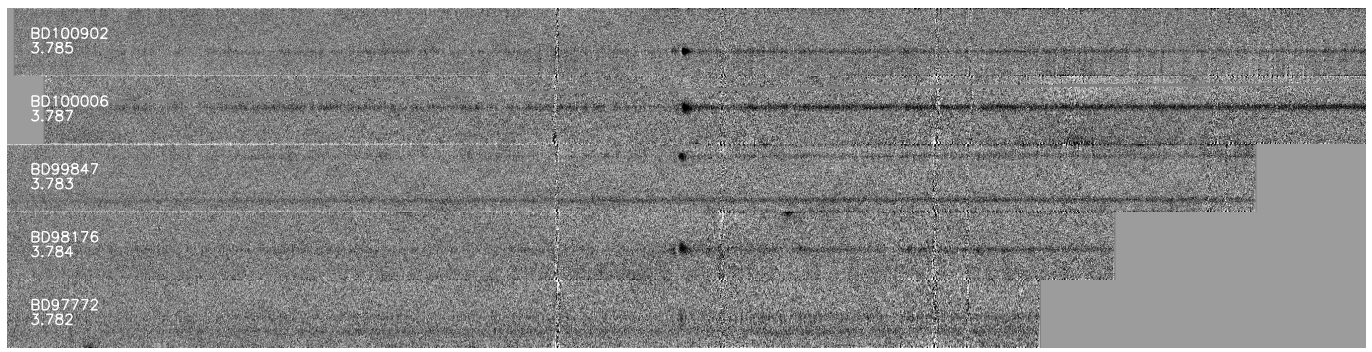

FIG. 12.- The two-dimensional DEIMOS spectra of the five galaxies at $z=3.78-3.79$ are shown. Each spectrum is shifted to align the spectra in the observed wavelengths. The presence of Ly $\alpha$ emission is evident in all cases. The close proximity in both angular and redshift space (see Figure 11) strongly suggest that these galaxies are physically associated. BD100902 and BD98176 have doubly-peaked Ly $\alpha$ emission.

(e.g., Doppler parameter, H I column density, the velocity distribution of the ISM).

Direct comparison with other known protoclusters at high redshift (e.g., Steidel et al. 2000; Ouchi et al. 2005; Daddi et al. 2009; Capak et al. 2011) is not trivial due to the varying depths and search methods of different surveys. The depth of the NDWFS data on which our selection of candidates is based allows us to probe down to $L \approx L^{*}$ at this redshift range, where the galaxy surface density is significantly lower $\left(<0.1 \mathrm{arcmin}^{-2}\right)$ than that of typical galaxies identified as protocluster mem- bers in other studies. For example, the 'GN20' protocluster members in the GOODS South field are identified down to $L \approx 0.1 L^{*}$ in their UV continuum level at the same redshift (Daddi et al. 2009). The surface density of $z \simeq 3.7$ photometric candidates in the GOODS South field is $\approx 9-10 \operatorname{arcmin}^{-2}$ (see Lee et al. 2012b).

The current spectroscopy only covers an $5^{\prime} \times 16^{\prime}$ of the area in the region, and we are unable to determine the physical extent of the structure. Deeper imaging and more spectroscopy over a wider area is crucially needed to better quantify the uniqueness of the structure and 
to directly compare the physical properties and evolutionary state of its constituents with those of the field galaxies (K.-S. Lee et al., in preparation).

\subsection{Galaxies with Multiple Interstellar Features}

Among the 41 spectroscopically confirmed LBGs at $3.2<z<4.6$, we have identified two sources, BD7645 and BD18449, that clearly exhibit two sets of interstellar absorption lines. The one-dimensional spectra of these galaxies are shown in Figure 13 where prominent interstellar absorption lines are marked at two different redshifts (cyan and red lines).

BD7645 is continuum-dominated with Ly $\alpha$ in absorption. The absorption features are very deep with nearly zero residual intensity in $\mathrm{Si}$ II $\lambda 1260$, C II $\lambda 1334, \mathrm{Si}$ IV $\lambda 1393$, and $\mathrm{C}$ IV $\lambda \lambda 1548,1550$ lines. The redshifts are 3.685 and 3.679 , implying the relative velocity of 380 $\mathrm{km} \mathrm{s}^{-1}$, the value comparable to that reported for $z \simeq$ $3-4$ galaxies (Shapley et al. 2003; Vanzella et al. 2009, see also 4.2 .

BD18449, exhibits a weak Ly $\alpha$ emission in combination with deep absorption trough. We measure the net EW to be $W_{0, \mathrm{Ly} \alpha}=-2.2 \AA$. The residual intensity is $\approx 0.5$, a half of that observed for the BD7645. The C II $\lambda 1334$ and Si II $\lambda 1526$ lines are at $z=3.744$, at rest with Ly $\alpha$, while O I+Si II $\lambda 1303$ and possibly with C IV $\lambda \lambda 1548,1550$ lines indicate an interstellar redshift of $z_{\mathrm{IS}}=3.726$. Interestingly, there is a hint of $\mathrm{N} \mathrm{IV]} \lambda 1486.5$, which is sometimes seen in emission but in this case, in absorption. There may be another component, if interpreted as a third O I+Si II $\lambda 1303$ blend, at an even higher velocity even though we cannot rule out the possibility of a chance absorber. The relative velocity of the two components in BD18449 implied by the two secure sets of interstellar redshifts is $1140 \mathrm{~km} \mathrm{~s}^{-1}$, the highest observed in our sample and twice larger than the median value $530 \pm 220$ $\mathrm{km} \mathrm{s}^{-1}$ (see 94.2 ).

Galaxies with multiple interstellar features are extremely rare with only a handful of reported cases; Among $\approx 3000$ galaxies at $z=1.5-3.5$ (LBGs, BX/BM: Steidel et al. 2003, 2004), there are 10 such sources identified (A. Shapley, private communication). The most well-known source is Q0000-D6 (D6, hereafter; Steidel et al. 2010, e.g., see their Figure 9), the most UVluminous galaxy $(\mathcal{R}=22.88)$ in their samples with the exception of MS 1512-cB58, which is highly magnified. Similar to our sources, D6 shows two distinct sets of interstellar absorption lines at $z_{\text {IS }}=2.9561,2.9635$, where the latter appears to be at the galaxy's systemic redshift (Steidel et al. 2010). D6 has a prominent Ly $\alpha$ emission which is redshifted with respect to the interstellar lines at $z_{\mathrm{Ly} \alpha}=2.9692$. Another luminous source, Q1623BX453 $(\mathcal{R}=23.38)$, shares similar characteristics to D6 $\left(z_{\mathrm{svs}}=2.1820, z_{\mathrm{IS}}=2.1724, z_{\mathrm{Ly} \alpha}=2.1838\right.$ : Law et al. 2007).

The simplest explanation of this phenomenon is that two galaxies in close proximity are mistakenly observed as a single source. In this scenario, the continuum luminosities of the two sources should be comparable in order to produce two visible sets of absorption line features. The NDWFS $I$ and $R$-band imaging data do not reveal multiple components for the two cases here; the surface brightness profile is consistent with a single isolated source in both cases. Thus, if the spectra are pro- duced by two distinct galaxies, they must lie within $0.5^{\prime \prime}$ (i.e., $3.5 \mathrm{kpc}$ in physical scale) of each other, perhaps in the last stage of merger. While we cannot rule out the possibility of witnessing two final-stage mergers, an alternate scenario would be that the spectrum is produced by two massive rotating clumps perhaps in the process of disk formation as proposed by Dekel et al. (2009). Highresolution imaging data will shed more light on the nature of these sources.

\section{DISCUSSION AND SUMMARY}

In this paper, we have investigated the physical properties of the most UV-luminous $\left(L \gtrsim L^{*}\right)$ star-forming galaxies at $z \simeq 3.7$ based on the rest-frame UV spectra of 41 galaxies obtained using the DEIMOS spectrograph at the W. M. Keck Observatory. The galaxies in our sample populate the exponential tail of the galaxy UV luminosity function, and have been under-represented in most previous studies due to their low surface density. They likely reside in more massive dark matter halos and are typically found to have larger stellar masses $(\gtrsim$ a few $\times 10^{10} M_{\odot}$ ) than their less-luminous counterparts (Lee et al. 2011). The relatively high $\mathrm{S} / \mathrm{N}$ spectra afforded by their optical brightness have allowed us to probe a variety of physical properties of individual galaxies as well as those of the population as a whole. Furthermore, we have discovered interesting classes of objects that are rarely found in lower-luminosity galaxies. These discoveries raise interesting questions about how similar the physical processes of galaxy formation are at different scales (i.e., environments, masses, luminosities). In what follows, we summarize our findings and discuss their physical implications in further detail.

The spectroscopy presented here validate the clean and efficient selection of photometric candidates, which we have identified over a much wider area, $5.3 \mathrm{deg}^{2}$ of the Boötes field, and have used to study the general properties of LBGs (Lee et al. 2011, 2012a). We estimate the contamination rate to be $\approx 11 \%$, mainly due to lowerredshift $(z<1)$ interlopers and high-redshift QSOs $(33$ and Figure 2). Even in the most pessimistic case where we assume all the galaxies that could not be identified spectroscopically are not at high redshift, the contamination rate is $28 \%$. The median luminosity of our spectroscopic sample is 2.1 times higher than previous samples of galaxies at comparable redshifts (Vanzella et al. 2009; Stark et al. 2010, see Figure 3).

We find that the $\operatorname{Ly} \alpha$ EW, i.e., the strength of $\operatorname{Ly} \alpha$ line emission relative to the UV continuum emission, in the galaxies in our sample is generally much weaker than that found for lower-luminosity galaxies in the literature (Vanzella et al. 2009; Stark et al. 2010). The median EW of our sample is $W_{0}=-1.2 \AA$, when we exclude galaxies that are likely not representative of our sample; these include galaxies at $z>4.2$ identified mainly via their strong Ly $\alpha$ emission despite their faint UV continuum, and five galaxies that belong to the galaxy overdensity at $z=3.78$. We find only one galaxy with $W_{0}>20 \AA$ among the 41 galaxies at $3.2<z<4.2$. Furthermore, at the brightest end $\left(M_{1700}<-22.2\right)$, there appears to be no galaxy with a positive Ly $\alpha \mathrm{EW}$. The trend of a decreasing number of Ly $\alpha$ emitting galaxies toward higher luminosity is clear in our data, supporting a changing physical parameter that affects the escape fraction of Ly $\alpha$ 


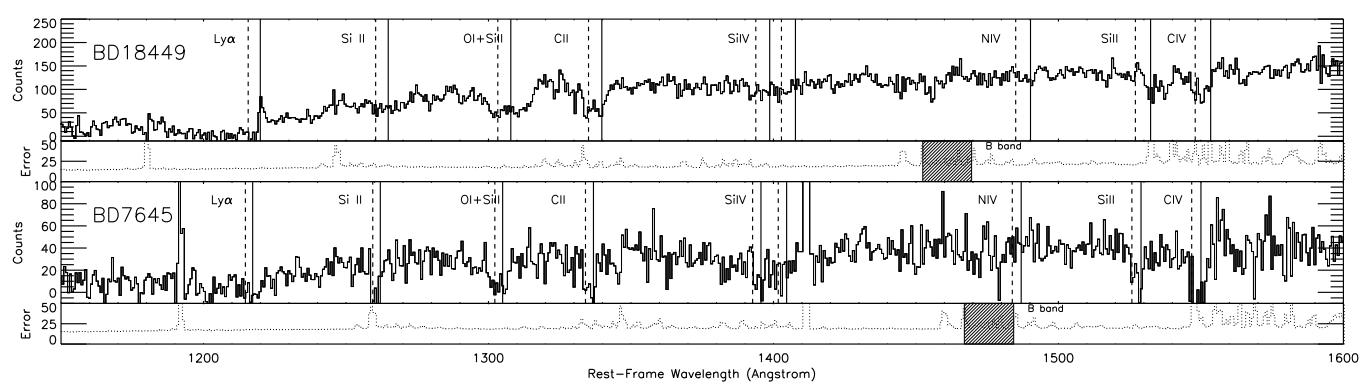

FIG. 13. - The spectra of the galaxies exhibiting multiple sets of interstellar absorption lines are shown. Both error spectra are in units of counts. The locations of prominent absorption lines are indicated by vertical lines at two different interstellar redshifts. The interstellar redshifts derived for BD7645 (bottom) are $z=3.685,3.679$, with the velocity offset $380 \mathrm{~km} \mathrm{~s}^{-1}$. On the other hand, BD18449 have $z_{\mathrm{IS}}=3.744,3.726$, where the former is at rest with the Ly $\alpha$ redshift. The velocity difference is $1140 \mathrm{~km} \mathrm{~s}^{-1}$, more than twice larger than the median value $(4.2$.

photons dramatically. It is important to quantify this dependence by increasing the number of spectroscopically observed luminous $\left(>3 L^{*}\right)$ galaxies.

The most straightforward explanation of this phenomenon is that the covering fraction of neutral gas in the ISM increases with UV luminosity. Such a picture is consistent with both the range of Ly $\alpha \mathrm{EW}$ observed at a given luminosity, and the gradual decline in the fraction of Ly $\alpha$-emitting galaxies towards higher luminosities. Our recent Herschel observations also support this view (Lee et al. 2012a): by comparing the UVderived extinction estimate (inferred by the UV continuum slope $\left.f_{\lambda} \propto \lambda^{\beta}\right)$ to a more direct measure of dust extinction (measured by the infrared-to-UV luminosity ratio), we find that the UV-based estimate under-predicts the true extinction by a factor of three for the most luminous galaxies (i.e., $M_{1700}<-22.2$ ). The two estimates can be brought into better agreement if the UV luminous galaxies follow a Small Magellanic Cloud (SMC)like dust reddening law (Bouchet et al. 1985) rather than the Calzetti law; the latter is often used as the standard assumption for high-redshift star-forming galaxies (Calzetti et al. 2000). The shallower slope for the extinction with wavelengths for the Calzetti law is generally interpreted as resulting from a clumpy ISM in the local starburst galaxies, which effectively allows more "blue" photons to escape without attenuation, compared to a more uniform coverage of the SMC-like ISM. Although the relative geometry of dust and neutral gas is unconstrained for high redshift galaxies, there is some evidence that they at least partially trace each other (Shapley et al. 2003). If the spatial distributions of dust and gas are largely decoupled, our interpretation would be invalid. We caution readers that our Herschel results are based on the photometric candidates, the majority of which are not spectroscopically verified. Even though the spectroscopy presented here seems to confirm the robustness of our sample with at most $28 \%$ of contamination, we cannot rule out the possibility that the results may be largely influenced by lower-redshift interlopers.

Another possibility is that the metallicity of the ISM increases with UV luminosity. The fact that interstellar absorption is generally stronger in higher-luminosity galaxies is qualitatively in agreement with this scenario. However, the line EWs in our observations are a combined product of the column density and velocity dispersion of the absorbing gas. Without knowledge of the intrinsic line profiles, it is difficult to disentangle these two factors and convert the measured EWs to the column density (i.e., relative abundances in the ISM). Locally, there is evidence that the velocity dispersion is larger in more UV-luminous and/or more reddened galaxies (Heckman et al. 1998), which is expected if more luminous galaxies have larger dynamical masses. Measurements of elemental abundance ratios based on higher$\mathrm{S} / \mathrm{N}$ spectra may be able to constrain the relative importance of these two factors in the future.

We investigated the relative velocity of the outflowing interstellar gas in our luminous LBG sample by measuring the difference between the redshifts of the interstellar absorption and Ly $\alpha$ emission lines, i.e., $\Delta V_{\text {out }} \equiv$ $V_{\mathrm{Ly} \alpha}-V_{\mathrm{abs}}$. We find that there is a correlation between the UV luminosity and $\Delta V_{\text {out }}$ in the sense that a higher-luminosity galaxy has a higher observed "maximum velocity" (see 4.2 and Figure 5). This implies that more UV-luminous galaxies have more powerful outflows which can potentially remove cold, neutral gas from the galaxy's potential well more effectively. Such a mechanism would have a profound effect on the subsequent formation and evolution of the galaxy. On the other hand, the gas in less powerful outflows may eventually trickle back into the galaxy, thereby only delaying the star-formation rather than preventing it entirely.

The observed correlation in our study is qualitatively in agreement with that found by Martin (2005) and Weiner et al. (2009) for local and intermediate-redshift star-forming galaxies. In contrast, observations of highredshift LBG samples by Steidel et al. (2010) suggest that the outflow velocity (defined as $V_{\text {abs }}$ ) is lower for galaxies with larger dynamical masses. It is possible that the large scatter in the observed velocity produced by random viewing angles has eroded any luminositydependent trend of the outflow velocity. The trend is subtle (e.g., Weiner et al. (2009) found $V_{\text {out }} \propto \mathrm{SFR}^{0.3}$ ) and the wider luminosity range probed by our current study may have helped identify the trend. We also find evidence that the outflow velocity is larger for the IRACdetected (and therefore presumably more massive) galaxies even though the low sensitivity of the current IRAC data could not provide reliable estimates of the masses for individual galaxies.

In order to study the average spectroscopic properties of the galaxies, we constructed a composite spectrum. The composite spectrum of galaxies exhibiting Ly $\alpha$ in absorption is compared to similar galaxies at $z \simeq 3$ (Shapley et al. 2003, 4.3.1 and Figure 7). The Ly $\alpha$ line 
morphology suggests that the column density and velocity distribution of the two samples are comparable. We find tentative evidence of enhanced emission in CIV and $\mathrm{N} \mathrm{V}$, both indicative of stellar winds, in our spectrum compared to the $z \simeq 3$ spectrum. If confirmed, the results would have interesting implications for the IMF and metallicity of galaxies at different luminosities. We also marginally detect $\mathrm{Ni}$ II, which is absent in the $z \simeq 3$ composite. In terms of the wind features and the relative strength of the Fe-peak element such as $\mathrm{Ni}$, our spectrum may be more similar to that of MS1512-cB58 than the $z \simeq 3$ spectrum. A possible interpretation of the differences is that more luminous galaxies (as represented by the composite spectrum of our sample) may have been forming stars longer and therefore has a higher relative enrichment in the Fe-peak elements (produced by intermediate-mass, longer-lived stars) than their lessluminous counterparts (the $z \simeq 3$ Shapley et al. composite).

We also compared the composite spectrum of galaxies with and without Ly $\alpha$ emission at comparable UV luminosities (\$4.3.2 and Figure 8). The interstellar line EWs are clearly stronger for the non-emitters, consistent with other studies in the literature. The trend likely reflects the combined effect from differing covering fractions and gas column densities of the two populations. However, we have found some evidence that there may also be intrinsic differences between the two populations. $\mathrm{Ni}$ II is detected in the non-emitter spectrum only, while $\mathrm{S} \mathrm{V}$ is seen in then emitter spectrum only. The results may imply that Ly $\alpha$-emitting galaxies generally have a 'younger' luminosity-weighted age than the non-emitters. The emerging picture is that the galaxy's age depends on both UV luminosity and Ly $\alpha$ EW. One simple explanation is that the covering fraction of neutral gas in the ISM increases with UV luminosity, and more luminous galaxies tend to be older.

Two independent observations provide circumstantial evidence to this scenario. First, the non-detection of He II emission in our composite, compared to its clear detection in the composite spectra of less-luminous $z \simeq 3$, $\simeq 4$ galaxies (Shapley et al. 2003; Jones et al. 2012), implies that W-R stars, which, as descendants of massive $\mathrm{O}$ stars, dominate the He II emission at the initial period $(\lesssim 20 \mathrm{Myr})$ of star-formation, are no longer present $(\$ 4.4$ and Figure 9). Second, the strengths of LIS absorption lines are clearly higher for more UV-luminous galaxies when the Ly $\alpha$ EW is fixed (\$4.5 and Figure 10 see also Jones et al. 2012). Such effects are likely contributed by a higher covering fraction, which determines the residual intensity of the spectrum, for UV-luminous galaxies although we cannot rule out the possibility that a higher column density and/or different kinematics of the gas are also responsible.

We have also discovered a possible large-scale structure at $z=3.78$ where five galaxies in our sample lie within $1 \mathrm{Mpc}$ (physical) from one another (\$5.1 and Figure 11). These galaxies appear to have enhanced Ly $\alpha$ emission, $\left\langle W_{0, \mathrm{Ly} \alpha}\right\rangle=14.2 \AA$, compared to $\left\langle W_{0, \mathrm{Ly} \alpha}\right\rangle=$ $-1.2 \AA$ for the field galaxies at the same redshift range (Figure 12 and 4). A similar trend was observed by Kuiper et al. (2012), but both observations are limited by small-number statistics. We are in the process of ob- taining a better census of the extent of this structure by surveying a much wider flanking region around these galaxies (K.-S. Lee, in preparation). More physical insights require more comprehensive, deeper coverage of the region, which is currently lacking. Within the hierarchical framework of galaxy formation, we argue that the discovery of a massive structure at high redshift is not at all surprising considering the unprecedented cosmic volume sampled by our candidates. Other wide-area surveys such as CFHTLS, SDSS Stripe 82, Dark Energy Survey (and, in the distant future, LSST) should be able to identify similar systems in large number, providing a clear view of how galaxy formation proceeded in the densest, most massive environments.

Of 41 galaxies in our sample, two show evidence of multiple interstellar components that are widely separated in the velocity space (\$5.2, Figure 13$)$; the relative velocities between the two components are $\approx 400 \mathrm{~km} \mathrm{~s}^{-1}$ for one case, and $\approx 1100 \mathrm{~km} \mathrm{~s}^{-1}$ for the other. Galaxies with multiple interstellar components are extremely rare $(<0.3 \%)$ in the existing studies at lower redshift $(z \simeq 2-3)$. Both galaxies appear to have a single component with a smooth surface brightness profile in the ground-based optical images, suggesting that if the interstellar features were produced by two distinct galaxies, they must be within $3 \mathrm{kpc}$ from each other, perhaps in the final stages of merging. While we cannot completely rule out the possibility of a chance observation of two such events based on the small-number statistics, we also consider an alternate physical mechanism: that the interstellar features originate from giant clumps produced by gravitational instability (e.g., Dekel et al. 2009). Regardless of their physical nature, the apparent high incidence rate of such systems in our sample of $L \gtrsim L^{*}$ galaxies warrants further investigation to obtain better statistics.

The data presented herein were obtained at the W.M. Keck Observatory, which is operated as a scientific partnership among the California Institute of Technology, the University of California and the National Aeronautics and Space Administration. The Observatory was made possible by the generous financial support of the W.M. Keck Foundation. We thank the staff of the W. M. Keck Observatory, in particular, Gregory Wirth. The analysis pipeline used to reduce the DEIMOS data was developed at UC Berkeley with support from NSF grant AST-0071048. KSL thanks Eros Vanzella and Tucker Jones for sharing their data and for insightful comments. The authors thank Alice Shapley for sharing her composite spectra and for useful discussions. The spectrum of MS1512 cB58 presented here was kindly provided by Chuck Steidel. This work is based in part on observations made with the Spitzer Space Telescope, which is operated by the Jet Propulsion Laboratory, California Institute of Technology. We are grateful to the expert assistance of the staff of Kitt Peak National Observatory where the optical and near-infrared observations of the NDWFS Boötes Field were obtained. The authors thank NOAO for supporting the NOAO Deep Wide-Field Survey. The authors also recognize and acknowledge the very significant cultural role and reverence that the summit of Mauna Kea has always had within the indigenous 
Hawaiian community.

\section{REFERENCES}

Adelberger, K. L., Steidel, C. C., Pettini, M., et al. 2005, ApJ, 619, 697

Adelberger, K. L., Steidel, C. C., Shapley, A. E., \& Pettini, M. 2003, ApJ, 584, 45

Ashby, M. L. N., Stern, D., Brodwin, et al. 2009, ApJ, 701, 428

Bouchet, P., Lequeux, J., Maurice, E., Prevot, L., \& PrevotBurnichon, M. L. 1985, A\&A, 149, 330

Bouwens, R. J., Illingworth, G. D., Blakeslee, J. P., Broadhurst, T. J., \& Franx, M. 2004, ApJ, 611, L1

Bouwens, R. J., Illingworth, G. D., Franx, M., et al., 2009, ApJ, 705, 936

Bouwens, R. J., Illingworth, G. D., Franx, M., \& Ford, H. 2007, ApJ, 670, 928

Bouwens, R. J., Illingworth, G. D., Oesch, P. A., et al. 2012, ApJ, 754,83

Calzetti, D., Armus, L., Bohlin, R. C., Kinney, A. L., Koornneef, J., \& Storchi-Bergmann, T. 2000, ApJ, 533, 682

Capak, P. L., Riechers, D., Scoville, N. Z., et al. 2011, Nature, 470 , 233

Chen, Y.-M., Tremonti, C. A., Heckman, T. M., Kauffmann, G., Weiner, B. J., Brinchmann, J., \& Wang, J. 2010, AJ, 140, 445

Cooper, M. C., Newman, J. A., Weiner, B. J. et al. 2008, MNRAS, 383,1058

Cooper, M. C., Newman, J. A., Davis, M., Finkbeiner, D. P., \& Gerke, B. F. 2012, Astrophysics Source Code Library, 3003

Curtis-Lake, E., McLure, R. J., Pearce, H. J., et al. 2012, MNRAS, 422,1425

Daddi, E., Dannerbauer, H., Stern, D., et al. 2009, ApJ, 694, 1517

Dekel, A., Sari, R., \& Ceverino, D. 2009, ApJ, 703, 785

Elbaz, D., Daddi, E., Le Borgne, D., et al. 2007, A\&A, 468, 33

Faber, S. M., Phillips, A. C., Kibrick, R. I., et al. 2003, in Society of Photo-Optical Instrumentation Engineers (SPIE) Conference Series, Vol. 4841, Society of Photo-Optical Instrumentation Engineers (SPIE) Conference Series, ed. M. Iye \& A. F. M. Moorwood, 1657-1669

Ferguson, H. C., Dickinson, M., Giavalisco, et al. 2004, ApJ, 600, L107

Finkelstein, S. L., Cohen, S. H., Moustakas, J. et al. 2011, ApJ, 733,117

Finkelstein, S. L., Papovich, C., Salmon, B., et al. 2012, ApJ, 756, 164

Finkelstein, S. L., Rhoads, J. E., Malhotra, S., \& Grogin, N. 2009, ApJ, 691, 465

Gawiser, E., van Dokkum, P. G., Herrera, et al. 2006, ApJS, 162,

Giavalisco, M. 2002, ARA\&A, 40, 579

Giavalisco, M., Dickinson, M., Ferguson, H. C., et al. 2004a, ApJ, 600, L103

Giavalisco, M., Ferguson, H. C., Koekemoer, A. M., et al. 2004b, ApJ, 600, L93

González, V., Labbé, I., Bouwens, R. J., Illingworth, G., Franx, M., \& Kriek, M. 2011, ApJ, 735, L34+

Heckman, T. M., Lehnert, M. D., Strickland, D. K., \& Armus, L. 2000, ApJS, 129, 493

Heckman, T. M., Robert, C., Leitherer, C., Garnett, D. R., \& van der Rydt, F. 1998, ApJ, 503, 646

Hildebrandt, H., Pielorz, J., Erben, T., Schneider, P., Eifler, T., Simon, P., \& Dietrich, J. P. 2007, A\&A, 462, 865

Hinshaw, G., Larson, D., Komatsu, E., et al. 2012, ArXiv e-prints (arXiv:1212.5226)

Jannuzi, B. T. \& Dey, A. 1999, in Astronomical Society of the Pacific Conference Series, 111

Jones, T., Stark, D. P., \& Ellis, R. S. 2012, ApJ, 751, 51

Komatsu, E., Smith, K. M., Dunkley, J., et al. 2011, ApJS, 192, 18

Kornei, K. A., Shapley, A. E., Erb, D. K., et al. 2010, ApJ, 711, 693

Kornei, K. A., Shapley, A. E., Martin, C. L., et al. 2012, ApJ, 758 135

Kuiper, E., Venemans, B. P., Hatch, N. A., Miley, G. K., \& Röttgering, H. J. A. 2012, MNRAS, 425, 801

Kulas, K. R., Shapley, A. E., Kollmeier, J. A., Zheng, Z., Steidel, C. C., \& Hainline, K. N. 2012, ApJ, 745, 33

Law, D. R., Steidel, C. C., Erb, D. K.et al. 2007, ApJ, 669, 929

Lee, K.-S., Giavalisco, M., Conroy, C., Wechsler, R. H., Ferguson, H. C., Somerville, R. S., Dickinson, M. E., \& Urry, C. M. 2009, ApJ, 695, 368
Lee, K.-S., Alberts, S., Atlee, D., Dey, A., Pope, A., Jannuzi, B. T., Reddy, N., \& Brown, M. J. I. 2012a, ApJ, 758, L31

Lee, K.-S., Dey, A., Reddy, N., et al., 2011, ApJ, 733, 99

Lee, K.-S., Ferguson, H. C., Wiklind, T., Dahlen, T., et al. 2012b, ApJ, 752, 66

Lee, K.-S., Giavalisco, M., Gnedin, O. Y., et al. 2006, ApJ, 642, 63

Madau, P. 1995, ApJ, 441, 18

Martin, C. L. 2005, ApJ, 621, 227

-. 2006, ApJ, 647, 222

Masters, D. \& Capak, P. 2011, PASP, 123, 638

Newman, J. A., Cooper, M. C., Davis, M., et al. 2012, ArXiv eprints

Oesch, P. A., Labbe, I., Bouwens, R. J., Illingworth, G. D., Gonzalez, V., Franx, M., Trenti, M., Holden, B. P., van Dokkum, P. G., \& Magee, D. 2012, ArXiv e-prints

Oke, J. B. \& Gunn, J. E. 1983, ApJ, 266, 713

Ouchi, M., Shimasaku, K., Okamura, et al. 2004a, ApJ, 611, 660

-. 2004b, ApJ, 611, 685

Ouchi, M. et al. 2005, ApJ, 620, L1

Papovich, C., Finkelstein, S. L., Ferguson, H. C., Lotz, J. M., \& Giavalisco, M. 2011, MNRAS, 412, 1123

Pentericci, L., Grazian, A., Fontana, A., Castellano, M., Giallongo, E., Salimbeni, S., \& Santini, P. 2009, A\&A, 494, 553

Pentericci, L., Grazian, A., Fontana, A., Salimbeni, S., Santini, P., de Santis, C., Gallozzi, S., \& Giallongo, E. 2007, A\&A, 471, 433

Pettini, M., Rix, S. A., Steidel, C. C., Adelberger, K. L., Hunt, M. P., \& Shapley, A. E. 2002, ApJ, 569, 742

Pettini, M., Steidel, C. C., Adelberger, K. L., Dickinson, M., \& Giavalisco, M. 2000, ApJ, 528, 96

Planck Collaboration, Ade, P. A. R., Aghanim, N., ArmitageCaplan, C. et al. 2013, ArXiv e-prints (arXiv:1303.5076)

Quider, A. M., Pettini, M., Shapley, A. E., \& Steidel, C. C. 2009, MNRAS, 398, 1263

Reddy, N., Dickinson, M., Elbaz, D., et al. 2012a, ApJ, 744, 154

Reddy, N. A., Pettini, M., Steidel, C. C., Shapley, A. E., Erb, D. K., \& Law, D. R. 2012b, ApJ, 754, 25

Reddy, N. A. \& Steidel, C. C. 2009, ApJ, 692, 778

Reddy, N. A., Steidel, C. C., Fadda, D., Yan, L., Pettini, M., Shapley, A. E., Erb, D. K., \& Adelberger, K. L. 2006, ApJ, 644, 792

Rupke, D. S., Veilleux, S., \& Sanders, D. B. 2005, ApJS, 160, 115

Schaerer, D. \& Vacca, W. D. 1998, ApJ, 497, 618

Schwartz, C. M. \& Martin, C. L. 2004, ApJ, 610, 201

Shapley, A. E., Steidel, C. C., Pettini, M., \& Adelberger, K. L. 2003, ApJ, 588, 65

Shapley, A. E. 2011, ARA\&A, 49, 525

Stark, D. P., Ellis, R. S., Bunker, A., Bundy, K., Targett, T., Benson, A., \& Lacy, M. 2009, ApJ, 697, 1493

Stark, D. P., Ellis, R. S., Chiu, K., Ouchi, M., \& Bunker, A. 2010, MNRAS, 408, 1628

Steidel, C. C., Adelberger, K. L., Giavalisco, M., Dickinson, M., \& Pettini, M. 1999, ApJ, 519, 1

Steidel, C. C., Adelberger, K. L., Shapley, A. E., Erb, D. K., Reddy, N. A., \& Pettini, M. 2005, ApJ, 626, 44

Steidel, C. C., Adelberger, K. L., Shapley, A. E., Pettini, M., Dickinson, M., \& Giavalisco, M. 2000, ApJ, 532, 170

-. 2003, ApJ, 592, 728

Steidel, C. C., Erb, D. K., Shapley, A. E., Pettini, M., Reddy, N., Bogosavljević, M., Rudie, G. C., \& Rakic, O. 2010, ApJ, 717, 289

Steidel, C. C., Pettini, M., \& Adelberger, K. L. 2001, ApJ, 546, 665

Steidel, C. C., Shapley, A. E., Pettini, M., Adelberger, K. L., Erb, D. K., Reddy, N. A., \& Hunt, M. P. 2004, ApJ, 604, 534

Vanzella, E., Cristiani, S., Dickinson, M., et al. 2006, A\&A, 454, 423

Vanzella, E., Giavalisco, M., Dickinson, M., et al., 2009, ApJ, 695, 1163

Verhamme, A., Schaerer, D., \& Maselli, A. 2006, A\&A, 460, 397

Weiner, B. J., Coil, A. L., Prochaska, J. X., et al. 2009, ApJ, 692, 187 